\title{
Finite Involution Semigroups with Infinite Irredundant Bases of Identities
}

\author{
Edmond W. H. Lee
}

Nova Southeastern University, edmond.lee@nova.edu

Follow this and additional works at: https://nsuworks.nova.edu/math_facarticles

Part of the Mathematics Commons

\section{NSUWorks Citation}

Lee, Edmond W. H., "Finite Involution Semigroups with Infinite Irredundant Bases of Identities" (2016). Mathematics Faculty Articles. 150.

https://nsuworks.nova.edu/math_facarticles/150 


\title{
Research Article
}

\section{Edmond W. H. Lee \\ Finite involution semigroups with infinite irredundant bases of identities}

\begin{abstract}
A basis of identities for an algebra is irredundant if each of its proper subsets fails to be a basis for the algebra. The first known examples of finite involution semigroups with infinite irredundant bases are exhibited. These involution semigroups satisfy several counterintuitive properties: their semigroup reducts do not have irredundant bases, they share reducts with some other finitely based involution semigroups, and they are direct products of finitely based involution semigroups.
\end{abstract}

Keywords: Semigroup, involution semigroup, identity, basis, irredundant basis, finite basis

MSC 2010: 20M05

Edmond W. H. Lee: Division of Math, Science, and Technology, Nova Southeastern University, 3301 College Avenue, Fort Lauderdale, Florida 33314, USA, e-mail: edmond.lee@nova.edu

Communicated by: Manfred Droste

\section{Introduction}

\subsection{Finite basis problem}

A basis for an algebra $A$ is a set of identities satisfied by $A$ that axiomatizes all identities of $A$. An algebra is finitely based if it has some finite basis. Finite groups [16], finite associative rings [7, 12], and finite Lie algebras [1] are all finitely based. However, this result does not hold for all finite algebras; there exist groupoids with as few as three elements that are not finitely based $[5,15]$. In general, the finite basis problem for finite algebras is undecidable [14], but the problem remains open when restricted to finite semigroups. The first examples of non-finitely based finite semigroups [17] were discovered in the 1960s. Since then, the finite basis problem for finite semigroups has been intensely investigated. For further information, refer to the survey by Volkov [19].

\subsection{Irredundant bases}

A basis for an algebra $A$ is irredundant if each of its proper subsets fails to be a basis for $A$. An algebra is irredundantly based if it has some irredundant basis. It is clear that any finitely based algebra is irredundantly based. As for non-finitely based finite algebras, there was initial hope that they all have irredundant bases, but this was refuted by subsequent examples of non-irredundantly based finite semigroups [13, 18]. On the other hand, there also exist finite semigroups with infinite irredundant bases [4]. Apart from these examples, not much is known about the irredundant basis property in general. The problem of deciding if a non-finitely based finite algebra is irredundantly based remains open.

\subsection{Involution semigroups}

Recall that an involution semigroup is a pair $\left(S,{ }^{*}\right)$, where the reduct $S$ is a semigroup and $x \mapsto x^{*}$ is a unary operation on $S$ such that

$$
\left(x^{*}\right)^{*}=x \text { and }(x y)^{*}=y^{*} x^{*} .
$$


Examples of involution semigroups include any group with inversion $x \mapsto x^{-1}$ and the multiplicative semigroup of all $n \times n$ matrices over any field with transposition $x \mapsto x^{\top}$. Compared with semigroups, even less is known about involution semigroups with respect to the irredundant basis property. Presently, only one finite involution semigroup is known to be non-irredundantly based [6]. Explicit examples of involution semigroups with infinite irredundant bases are unavailable. The objective of the present article is to exhibit the first examples of such finite involution semigroups. These new examples, together with recently established results $[9,10]$, demonstrate that an involution semigroup and its reduct can satisfy very contrasting equational properties.

\subsection{Known results}

The main examples of the present article are constructed from the cyclic group

$$
\mathbb{Z}_{n}=\left\langle g \mid g^{n}=1\right\rangle=\left\{1, g, g^{2}, \ldots, g^{n-1}\right\}
$$

of order $n \geq 1$ and the $\mathscr{J}$-trivial semigroup

$$
L=\left\langle e, f \mid e^{2}=e, f^{2}=f, e f e=0\right\rangle=\{0, e, f, e f, f e, f e f\}
$$

of order six. The multiplication table of $L$ is given as follows.

\begin{tabular}{rrrrrrr}
\hline $\boldsymbol{L}$ & $\mathbf{0}$ & $\boldsymbol{f e f}$ & $\boldsymbol{e f}$ & $\boldsymbol{f e}$ & $\boldsymbol{e}$ & $\boldsymbol{f}$ \\
$\mathbf{0}$ & 0 & 0 & 0 & 0 & 0 & 0 \\
$f e f$ & 0 & 0 & 0 & 0 & 0 & $f e f$ \\
$\boldsymbol{e f}$ & 0 & 0 & 0 & 0 & 0 & $e f$ \\
$f \boldsymbol{e}$ & 0 & 0 & $f e f$ & 0 & $f e$ & $f e f$ \\
$\boldsymbol{e}$ & 0 & 0 & $e f$ & 0 & $e$ & $e f$ \\
$\boldsymbol{f}$ & 0 & $f e f$ & $f e f$ & $f e$ & $f e$ & $f$ \\
\hline
\end{tabular}

Up to isomorphism, the semigroup $L$ is one of only four non-finitely based semigroups of order six [11]. The direct product $L \times \mathbb{Z}_{n}$ of $L$ and $\mathbb{Z}_{n}$ is a non-finitely based semigroup [8], and this result was recently generalized.

Theorem 1.1 ([9, Theorem 1.3]). For each $n \geq 1$, the non-finitely based semigroup $L \times \mathbb{Z}_{n}$ is non-irredundantly based.

For each $n \geq 1$, let $\mathrm{sq}(n)$ denote the set of all square roots of unity modulo $n$, that is,

$$
\mathrm{sq}(n)=\left\{\mathbb{R} \in\{1,2, \ldots, n\}: \mathbb{R}^{2} \equiv 1(\bmod n)\right\} .
$$

It is routinely checked that for each $\mathbb{R} \in \mathrm{sq}(n)$,

$$
\mathbf{Z}_{n}^{\mathbb{R}}=\left(\mathbb{Z}_{n},{ }^{\mathbb{R}}\right) \quad \text { with } \quad x \mapsto x^{\mathbb{R}}
$$

is an involution semigroup. Conversely, any involution semigroup with reduct $\mathbb{Z}_{n}$ is isomorphic to some $\mathbf{Z}_{n}^{\mathbb{R}}$, so the number of such involution semigroups is

$$
|\operatorname{sq}(n)|= \begin{cases}2^{\omega(n)+1} & \text { if } n \equiv 0(\bmod 8) \\ 2^{\omega(n)-1} & \text { if } n \equiv \pm 2(\bmod 8) \\ 2^{\omega(n)} & \text { otherwise }\end{cases}
$$

where $\omega(n)$ is the number of distinct prime factors of $n$ ([3]). In particular, the inversion operation $x \mapsto x^{-1}$ in $\mathbb{Z}_{n}$ coincides with $x \mapsto x^{n-1}$.

As for the semigroup $L$, it is the reduct of the involution semigroup

$$
\mathbf{L}=\left(L,{ }^{\lambda}\right),
$$


where ${ }^{\lambda}$ interchanges ef and $f e$ but fixes all other elements of $L$. In fact, up to isomorphism, $\mathrm{L}$ is the unique involution semigroup with reduct $L$. Hence, the direct product of the involution semigroups $\mathbf{L}$ and $\mathbf{Z}_{n}^{\mathbb{R}}$ is

$$
\mathbf{L} \times \mathbf{Z}_{n}^{\mathbb{R}}=\left(L \times \mathbb{Z}_{n},{ }^{*}\right) \quad \text { with } \quad(x, y)^{*}=\left(x^{\lambda}, y^{\mathbb{R}}\right) .
$$

Consequently, up to isomorphism, the number of involution semigroups with reduct $L \times \mathbb{Z}_{n}$ is precisely $|\operatorname{sq}(n)|$.

The finite basis property of the involution semigroup $\mathbf{L} \times \mathbf{Z}_{n}^{\mathbb{R}}$, when $\mathbb{R}=1$, was recently established.

Theorem 1.2 ([10, Theorem 1.2]). For each $n \geq 1$, the involution semigroup $\mathbf{L} \times \mathbf{Z}_{n}^{1}$ is finitely based and so is irredundantly based.

\subsection{Main result}

In view of Theorem 1.2, what remains to be investigated is the involution semigroup $\mathbf{L} \times \mathbf{Z}_{n}^{\mathbb{R}}$ with $\mathbb{R} \in \operatorname{sq}(n) \backslash\{1\}$.

Theorem 1.3. For each $n \geq 1$ and $\mathbb{R} \in \mathrm{sq}(n) \backslash\{1\}$, the involution semigroup $\mathrm{L} \times \mathbf{Z}_{n}^{\mathbb{R}}$ has an infinite irredundant basis and so is non-finitely based.

The results of Theorems 1.1-1.3 are summarized in Table 1.

\begin{tabular}{rrrr}
\hline & $L \times \mathbb{Z}_{n}$ & $\mathrm{~L} \times \mathbf{Z}_{n}^{1}$ & $\mathrm{~L} \times \mathbf{Z}_{n}^{\mathbb{R}}, \mathbb{R} \in \mathbf{s q}(n) \backslash\{\mathbf{1}\}$ \\
\hline Finitely based & No & Yes & No \\
Irredundantly based & No & Yes & Yes \\
\hline
\end{tabular}

Table 1. Summary of Theorems 1.1-1.3.

Corollary 1.4. For each $n \geq 1$ and $\mathbb{R} \in \mathrm{sq}(n)$, the involution semigroup $\mathbf{L} \times \mathbf{Z}_{n}^{\mathbb{R}}$ is

(i) irredundantly based,

(ii) finitely based if and only if $\mathbb{R}=1$.

Corollary 1.5. There exist finite examples of

(i) involution semigroups with infinite irredundant bases such that their reducts do not have such bases,

(ii) non-finitely based involution semigroups sharing the same reducts with finitely based involution semigroups,

(iii) non-finitely based involution semigroups that are direct products of finitely based involution semigroups.

Proof. Refer to Table 1 for examples of parts (i) and (ii). As for part (iii), the involution semigroup $\mathbf{L} \times \mathbf{Z}_{n}^{n-1}$ is non-finitely based for all $n \geq 3$, while the involution semigroups $\mathbf{L}$ and $\mathbf{Z}_{n}^{n-1}$ are finitely based.

Since all finitely based algebras are irredundantly based, no involution semigroup can provide a ( $\left(\begin{array}{l}\text { Yes } \\ \text { No }\end{array}\right)$-column in Table 1. Therefore, this table exhibits the most complicated scenario for a non-irredundantly based finite semigroup $S$ in the following sense: there exist involution semigroups $\left(S,{ }^{*}\right)$ and $\left(S,{ }^{\dagger}\right)$ with reduct $S$ such that no two of $S,\left(S,{ }^{*}\right)$, and $\left(S,{ }^{\dagger}\right)$ simultaneously satisfy the same finite basis property and irredundant basis property. It is of interest to know if the most complicated scenario exists for an irredundantly based finite semigroup.

Question 1.6. Is there an irredundantly based finite semigroup $S$, being the reduct of involution semigroups $\left(S,{ }^{*}\right)$ and $\left(S,{ }^{\dagger}\right)$, such that their finite basis property and irredundant basis property are described in one of the following tables?

\begin{tabular}{rrrr}
\hline & $S$ & $\left(S,{ }^{*}\right)$ & $\left(S,{ }^{\dagger}\right)$ \\
\hline Finitely based & Yes & No & No \\
Irredundantly based & Yes & Yes & No \\
\hline
\end{tabular}

\begin{tabular}{rrrr}
\hline & $S$ & $\left(S,{ }^{*}\right)$ & $\left(S,{ }^{\dagger}\right)$ \\
\hline Finitely based & No & Yes & No \\
Irredundantly based & Yes & Yes & No \\
\hline
\end{tabular}




\subsection{Smaller examples}

Although the order $6 n$ of the irredundantly based involution semigroup $\mathbf{L} \times \mathbf{Z}_{n}^{\mathbb{R}}$ in Theorem 1.3 can be considered small, smaller examples are available. For each $n \geq 1$, the subset

$$
L_{n}=(L \times\{1\}) \cup\left(\{0\} \times \mathbb{Z}_{n}\right)
$$

of $L \times \mathbb{Z}_{n}$ has $n+5$ elements. It is routinely verified that $L_{n}$ is a subsemigroup of $L \times \mathbb{Z}_{n}$ that is closed under the unary operation $(x, y)^{*} \mapsto\left(x^{\lambda}, y^{\mathbb{R}}\right)$ of $\mathbf{L} \times \mathbf{Z}_{n}^{\mathbb{R}}$. Hence, $\mathbf{L}_{n}^{\mathbb{R}}=\left(L_{n},{ }^{*}\right)$ is an involution subsemigroup of $\mathbf{L} \times \mathbf{Z}_{n}^{\mathbb{R}}$ of order $n+5$.

Lemma 1.7. For each $n \geq 1$, the involution semigroups $\mathbf{L}_{n}^{\mathbb{R}}$ and $\mathbf{L} \times \mathbf{Z}_{n}^{\mathbb{R}}$ satisfy the same identities.

Proof. The identities satisfied by $\mathbf{L} \times \mathbf{Z}_{n}^{\mathbb{R}}$ are vacuously satisfied by its involution subsemigroup $\mathbf{L}_{n}^{\mathbb{R}}$. Conversely, since

$$
\mathbf{L} \times \mathbf{Z}_{n}^{\mathbb{R}}=\left(L,{ }^{\lambda}\right) \times\left(\mathbb{Z}_{n},{ }^{\mathbb{R}}\right) \cong\left(L \times\{1\},{ }^{*}\right) \times\left(\{0\} \times \mathbb{Z}_{n},{ }^{*}\right) \subseteq \mathbf{L}_{n}^{\mathbb{R}} \times \mathbf{L}_{n}^{\mathbb{R}},
$$

the identities satisfied by $\mathbf{L}_{n}^{\mathbb{R}}$ are also satisfied by $\mathbf{L} \times \mathbf{Z}_{n}^{\mathbb{R}}$.

Consequently, the involution semigroup $\mathrm{L}_{3}^{2}$ of order eight has an infinite irredundant basis; it is presently the smallest known example. As for semigroups with infinite irredundant bases, the smallest example currently known is of order nine [4].

Question 1.8. What is the smallest possible order of a semigroup or involution semigroup with infinite irredundant basis?

\subsection{Organization}

There are seven sections in the present article. Notation and background material are given in Section 2. Sections 3-6 are devoted to establishing an explicit infinite basis for the involution semigroup $\mathbf{L} \times \mathbf{Z}_{n}^{\mathbb{R}}$. This explicit basis is then shown in Section 7 to contain an infinite irredundant basis for $\mathbf{L} \times \mathbf{Z}_{n}^{\mathbb{R}}$. Consequently, the proof of Theorem 1.3 is complete.

\section{Preliminaries}

The free involution semigroup over a countably infinite alphabet $\mathscr{A}$ is the free semigroup $\left(\mathscr{A}^{\cup} \mathscr{A}^{*}\right)^{+}$over the disjoint union of $\mathscr{A}$ and $\mathscr{A}^{*}=\left\{x^{*}: x \in \mathscr{A}\right\}$, with endowed unary operation ${ }^{*}$ defined by $\left(x^{*}\right)^{*}=x$ for all $x \in \mathscr{A}$ and

$$
\left(x_{1} x_{2} \cdots x_{m}\right)^{*}=x_{m}^{*} x_{m-1}^{*} \cdots x_{1}^{*}
$$

for all $x_{1}, x_{2}, \ldots, x_{m} \in \mathscr{A} \cup \mathscr{A}^{*}$. Elements of $\mathscr{A} \cup \mathscr{A}^{*}$ are called letters while elements of $\left(\mathscr{A} \cup \mathscr{A}^{*}\right)^{+} \cup\{\varnothing\}$ are called words. Words in $\mathscr{A}^{+} \cup\{\varnothing\}$ are called plain words. A word that is also a single letter is called a singleton. Any word $\mathbf{w} \in\left(\mathscr{A} \cup \mathscr{A}^{*}\right)^{+}$can be written in the form

$$
\mathbf{w}=x_{1}^{\circledast_{1}} x_{2}^{\circledast_{2}} \cdots x_{m}^{\circledast_{m}},
$$

where $x_{1}, x_{2}, \ldots, x_{m} \in \mathscr{A}$ and $\circledast_{1}, \circledast_{2}, \ldots, \circledast_{m} \in\{1, *\}$; the plain projection of such a word is the plain word

$$
\overline{\mathbf{w}}=x_{1} x_{2} \cdots x_{m}
$$

The first and last letters that occur in a word $\mathbf{w}$ are denoted by $\triangleright \mathbf{w}$ and $\mathbf{w} \triangleleft$, respectively. The number of times that a letter $x$ occurs in a word $\mathbf{w}$ is denoted $b y \operatorname{occ}(x, \mathbf{w})$. The content of a word $\mathbf{w}$ is defined to be the set

$$
\operatorname{con}(\overline{\mathbf{w}})=\{x \in \mathscr{A}: \operatorname{occ}(x, \overline{\mathbf{w}}) \geq 1\}
$$


this set is partitioned into the sets

$$
\operatorname{sim}(\overline{\mathbf{w}})=\{x \in \mathscr{A}: \operatorname{occ}(x, \overline{\mathbf{w}})=1\} \quad \text { and } \operatorname{non}(\overline{\mathbf{w}})=\{x \in \mathscr{A}: \operatorname{occ}(x, \overline{\mathbf{w}}) \geq 2\} .
$$

The letters in $\operatorname{sim}(\overline{\mathbf{w}})$ are said to be simple in $\mathbf{w}$ while the letters in non $(\overline{\mathbf{w}})$ are said to be non-simple in $\mathbf{w}$. A word $\mathbf{w}$ is non-simple if some letter is non-simple in it, that is, $\operatorname{non}(\overline{\mathbf{w}}) \neq \varnothing$. Two words $\mathbf{w}$ and $\mathbf{w}^{\prime}$ are disjoint if $\operatorname{con}(\overline{\mathbf{w}}) \cap \operatorname{con}\left(\overline{\mathbf{w}^{\prime}}\right)=\varnothing$.

An identity is written as $\mathbf{w} \approx \mathbf{w}^{\prime}$, where $\mathbf{w}$ and $\mathbf{w}^{\prime}$ are words. An involution semigroup $\mathbf{S}=\left(S,{ }^{*}\right)$ satisfies an identity $\mathbf{w} \approx \mathbf{w}^{\prime}$ if, for any substitution $\varphi: \mathscr{A} \rightarrow S$, the elements $\mathbf{w} \varphi$ and $\mathbf{w}^{\prime} \varphi$ of $S$ are equal. An identity $\mathbf{w} \approx \mathbf{w}^{\prime}$ is deducible from a set $\Sigma$ of identities, written $\Sigma \vdash \mathbf{w} \approx \mathbf{w}^{\prime}$, if every involution semigroup that satisfies all identities in $\Sigma$, satisfies $\mathbf{w} \approx \mathbf{w}^{\prime}$ as well. Two sets of identities $\Sigma_{1}$ and $\Sigma_{2}$ are equivalent, written $\Sigma_{1} \sim \Sigma_{2}$, if the deductions $\Sigma_{1} \vdash \Sigma_{2}$ and $\Sigma_{2} \vdash \Sigma_{1}$ hold. The set of all identities satisfied by an involution semigroup $S$ is denoted by id(S). A subset $\Sigma$ of id(S) is a basis for $\mathbf{S}$ if $\Sigma \vdash \mathrm{id}(\mathbf{S})$. An involution semigroup is finitely based if it possesses a finite basis.

Lemma 2.1 ([10, Lemma 2.1]). If $\mathbf{w} \approx \mathbf{w}^{\prime} \in \operatorname{id}(\mathbf{L})$, then $\operatorname{sim}(\overline{\mathbf{w}})=\operatorname{sim}\left(\overline{\mathbf{w}^{\prime}}\right)$ and $\operatorname{non}(\overline{\mathbf{w}})=\operatorname{non}\left(\overline{\mathbf{w}^{\prime}}\right)$.

Lemma 2.2 ([10, Lemma 2.2]). For any $\mathbf{w}, \mathbf{w}^{\prime} \in \mathscr{A}^{+}$, the involution semigroup $\mathbf{Z}_{n}^{\mathbb{R}}$ satisfies the identity $\mathbf{w} \approx \mathbf{w}^{\prime}$ if and only if $\operatorname{occ}(x, \overline{\mathbf{w}}) \equiv \operatorname{occ}\left(x, \overline{\mathbf{w}^{\prime}}\right)(\bmod n)$ for all $x \in \mathscr{A}$.

\section{An explicit basis for $\mathrm{L} \times \mathrm{Z}_{n}^{\mathbb{R}}$}

If $n \in\{1,2\}$, then $\mathrm{sq}(n) \backslash\{1\}=\varnothing$, so that $\mathbf{L} \times \mathbf{Z}_{n}^{\mathbb{R}}=\mathbf{L} \times \mathbf{Z}_{n}^{1}$ is finitely based by Theorem 1.2. If $n \geq 3$, then $n-1 \in \mathrm{sq}(n) \backslash\{1\}$, so that $\mathrm{sq}(n) \backslash\{1\} \neq \varnothing$. Therefore, for the remainder of the article, assume that $n \geq 3$ and $\mathbb{R} \in \mathrm{sq}(n) \backslash\{1\}$ are fixed. Furthermore, the axioms

$$
(x y) z \approx x(y z), \quad\left(x^{*}\right)^{*} \approx x, \quad(x y)^{*} \approx y^{*} x^{*}
$$

of involution semigroups are assumed to hold in all deductions. In other words, for any set $\Sigma$ of identities and any identity $\mathbf{w} \approx \mathbf{w}^{\prime}$, the deduction $\{($ inv $)\} \cup \Sigma \vdash \mathbf{w} \approx \mathbf{w}^{\prime}$ is simply written as $\Sigma \vdash \mathbf{w} \approx \mathbf{w}^{\prime}$.

Theorem 3.1. The identities

$$
\begin{aligned}
& x^{n+2} \approx x^{2}, \quad x^{n+1} y x \approx x y x, \quad x y x^{n+1} \approx x y x, \\
& x^{2} y x \approx x y x^{2}, \\
& x y x z x \approx x z x y x \text {, } \\
& \left(x^{n}\right)^{*} \approx x^{n}, \\
& x^{*} \mathbf{h}_{1} x^{*} \approx x^{\mathbb{R}} \mathbf{h}_{1} x^{\mathbb{R}}, \\
& x^{*} \mathbf{h}_{1} x \mathbf{h}_{2} x \approx x^{\mathbb{R}} \mathbf{h}_{1} x \mathbf{h}_{2} x \text {, } \\
& x \mathbf{h}_{1} x^{*} \mathbf{h}_{2} x \approx x \mathbf{h}_{1} x^{\mathbb{R}} \mathbf{h}_{2} x \text {, } \\
& x \mathbf{h}_{1} x \mathbf{h}_{2} x^{*} \approx x \mathbf{h}_{1} x \mathbf{h}_{2} x^{\mathbb{R}}, \\
& x^{*} \mathbf{h}_{1} y^{\oplus_{1}} \mathbf{h}_{2} x^{\circledast_{2}} \mathbf{h}_{3} y^{\oplus_{3}} \approx x^{\mathbb{R}} \mathbf{h}_{1} y^{\oplus_{1}} \mathbf{h}_{2} x^{\oplus_{2}} \mathbf{h}_{3} y^{\oplus_{3}} \text {, } \\
& x^{\otimes_{1}} \mathbf{h}_{1} y^{*} \mathbf{h}_{2} x^{\otimes_{2}} \mathbf{h}_{3} y^{\otimes_{3}} \approx x^{\circledast_{1}} \mathbf{h}_{1} y^{\mathbb{R}} \mathbf{h}_{2} x^{\otimes_{2}} \mathbf{h}_{3} y^{\otimes_{3}} \text {, } \\
& x^{\oplus_{1}} \mathbf{h}_{1} y^{\oplus_{2}} \mathbf{h}_{2} x^{*} \mathbf{h}_{3} y^{\oplus_{3}} \approx x^{\circledast_{1}} \mathbf{h}_{1} y^{\oplus_{2}} \mathbf{h}_{2} x^{\mathbb{R}} \mathbf{h}_{3} y^{\oplus_{3}} \text {, } \\
& x^{\otimes_{1}} \mathbf{h}_{1} y^{\otimes_{2}} \mathbf{h}_{2} x^{\otimes_{3}} \mathbf{h}_{3} y^{*} \approx x^{\otimes_{1}} \mathbf{h}_{1} y^{\otimes_{2}} \mathbf{h}_{2} x^{\circledast_{3}} \mathbf{h}_{3} y^{\mathbb{R}} \text {, } \\
& \left(\mathbf{h}_{1} x y \mathbf{h}_{2}\right)^{n+1} \approx\left(\mathbf{h}_{1} y x \mathbf{h}_{2}\right)^{n+1}, \\
& x^{p} \mathbf{h}_{1} y^{q} \mathbf{h}_{2} x \mathbf{h}_{3} y \approx y^{q} \mathbf{h}_{1} x^{p} \mathbf{h}_{2} y \mathbf{h}_{3} x, \quad 1 \leq p, q \leq n, \\
& x \mathbf{h}_{1} y \mathbf{h}_{2} x \mathbf{h}_{3} y \approx x y^{n} \mathbf{h}_{1} y \mathbf{h}_{2} x \mathbf{h}_{3} y \text {, } \\
& x \mathbf{h}_{1} y \mathbf{h}_{2} x \mathbf{h}_{3} y \approx y^{n} x \mathbf{h}_{1} y \mathbf{h}_{2} x \mathbf{h}_{3} y,
\end{aligned}
$$




$$
\begin{aligned}
x \mathbf{h}_{1} y \mathbf{h}_{2} x \mathbf{h}_{3} y & \approx x \mathbf{h}_{1} x^{n} y \mathbf{h}_{2} x \mathbf{h}_{3} y, \\
x \mathbf{h}_{1} y \mathbf{h}_{2} x \mathbf{h}_{3} y & \approx x \mathbf{h}_{1} y x^{n} \mathbf{h}_{2} x \mathbf{h}_{3} y, \\
x \mathbf{h}_{1} y \mathbf{h}_{2} x \mathbf{h}_{3} y & \approx x \mathbf{h}_{1} y \mathbf{h}_{2} x y^{n} \mathbf{h}_{3} y, \\
x \mathbf{h}_{1} y \mathbf{h}_{2} x \mathbf{h}_{3} y & \approx x \mathbf{h}_{1} y \mathbf{h}_{2} y^{n} x \mathbf{h}_{3} y, \\
x \mathbf{h}_{1} y \mathbf{h}_{2} x \mathbf{h}_{3} y & \approx x \mathbf{h}_{1} y \mathbf{h}_{2} x \mathbf{h}_{3} x^{n} y \\
x \mathbf{h}_{1} y \mathbf{h}_{2} x \mathbf{h}_{3} y & \approx x \mathbf{h}_{1} y \mathbf{h}_{2} x \mathbf{h}_{3} y x^{n}, \\
x\left(\prod_{i=1}^{m}\left(y_{i} \mathbf{h}_{i} y_{i}^{*}\right)\right) x^{*} & \approx x\left(\prod_{i=m}^{1}\left(y_{i} \mathbf{h}_{i} y_{i}^{*}\right)\right) x^{*}, \quad m \geq 2,
\end{aligned}
$$

where $\mathbf{h}_{i} \in\left\{\varnothing, h_{i}\right\}$ and $\circledast_{i} \in\{1, *\}$ constitute a basis for the involution semigroup $\mathbf{L} \times \mathbf{Z}_{n}^{\mathbb{R}}$.

Lemma 3.2. The involution semigroup $\mathbf{L} \times \mathbf{Z}_{n}^{\mathbb{R}}$ satisfies identities (3.1).

Proof. It follows from [10, Lemma 3.2] that the involution semigroup L satisfies identities (3.1). Since the group $\mathbf{Z}_{n}^{\mathbb{R}}$ satisfies the identities $x^{n} \approx 1, x^{*} \approx x^{\mathbb{R}}$, and $x y \approx y x$, it is routinely shown that it also satisfies identities (3.1).

Some restrictions on the identities satisfied by the involution semigroup $\mathbf{L} \times \mathbf{Z}_{n}^{\mathbb{R}}$ are established in Sections 4 and 5. The proof of Theorem 3.1 is then given in Section 6. The basis (3.1) is shown in Section 7 to contain an infinite irredundant basis for $\mathbf{L} \times \mathbf{Z}_{n}^{\mathbb{R}}$, hence completing the proof of Theorem 1.3.

\section{Connected identities and sandwich identities}

Recall that two words $\mathbf{w}_{1}$ and $\mathbf{w}_{2}$ are disjoint if $\operatorname{con}\left(\overline{\mathbf{w}_{1}}\right) \cap \operatorname{con}\left(\overline{\mathbf{w}_{2}}\right)=\varnothing$. A non-simple word is connected if it cannot be decomposed into a product of two disjoint nonempty words. In other words, a non-simple word $\mathbf{w}$ is connected if whenever $\mathbf{w}=\mathbf{w}_{1} \mathbf{w}_{2}$ for some $\mathbf{w}_{1}, \mathbf{w}_{2} \in\left(\mathscr{A} \cup \mathscr{A}^{*}\right)^{+}$, then $\operatorname{con}\left(\overline{\mathbf{w}_{1}}\right) \cap \operatorname{con}\left(\overline{\mathbf{w}_{2}}\right) \neq \varnothing$. An identity $\mathbf{w} \approx \mathbf{w}^{\prime}$ is connected if the words $\mathbf{w}$ and $\mathbf{w}^{\prime}$ are connected. It is shown in Section 4.1 that the involution semigroup $\mathbf{L} \times \mathbf{Z}_{n}^{\mathbb{R}}$ possesses a basis that consists entirely of connected identities. In Section 4.2, a special kind of connected identities, called sandwich identities, is introduced. It is shown in Lemma 4.4 that the involution semigroup $\mathbf{L} \times \mathbf{Z}_{n}^{\mathbb{R}}$ possesses a basis that consists of (3.1) and sandwich identities.

The results in the present section, together with those from Section 5, are required in Section 6 in the proof of Theorem 3.1.

\subsection{Connected identities}

Lemma 4.1. Let $\mathbf{w} \approx \mathbf{w}^{\prime} \in \mathrm{id}\left(\mathbf{L} \times \mathbf{Z}_{n}^{\mathbb{R}}\right)$. Suppose that $\mathbf{w}=\mathbf{w}_{1} \mathbf{w}_{2}$ for some disjoint words $\mathbf{w}_{1}, \mathbf{w}_{2} \in\left(\mathscr{A} \cup \mathscr{A}^{*}\right)^{+}$. Then, $\mathbf{w}^{\prime}=\mathbf{w}_{1}^{\prime} \mathbf{w}_{2}^{\prime}$ for some disjoint words $\mathbf{w}_{1}^{\prime}, \mathbf{w}_{2}^{\prime} \in\left(\mathscr{A} \cup \mathscr{A}^{*}\right)^{+}$such that $\mathbf{w}_{1} \approx \mathbf{w}_{1}^{\prime}, \mathbf{w}_{2} \approx \mathbf{w}_{2}^{\prime} \in \mathrm{id}\left(\mathbf{L} \times \mathbf{Z}_{n}^{\mathbb{R}}\right)$. Consequently, $\mathbf{w}$ is connected if and only if $\mathbf{w}^{\prime}$ is connected.

Proof. This result has been established for the case $\mathbb{R}=1$ ([10, Lemma 4.1]), but its proof is independent of the value of $\mathbb{R}$. Hence, the present lemma holds.

Any word $\mathbf{u} \in\left(\mathscr{A} \cup \mathscr{A}^{*}\right)^{+}$can be decomposed as

$$
\mathbf{u}=\prod_{i=1}^{m} \mathbf{p}_{i}
$$

where $\mathbf{p}_{1}, \mathbf{p}_{2}, \ldots, \mathbf{p}_{m} \in\left(\mathscr{A} \cup \mathscr{A}^{*}\right)^{+}$are pairwise disjoint words each of which is either a singleton or a connected word. It is easily seen that this decomposition of $\mathbf{u}$, called the natural decomposition of $\mathbf{u}$, is unique.

Lemma 4.2. The involution semigroup $\mathbf{L} \times \mathbf{Z}_{n}^{\mathbb{R}}$ possesses a basis that consists entirely of connected identities. 
Proof. Let $\mathbf{u} \approx \mathbf{u}^{\prime}$ be any identity from a basis $\Sigma$ for $\mathbf{L} \times \mathbf{Z}_{n}^{\mathbb{R}}$. Suppose that $\mathbf{u}=\prod_{i=1}^{m} \mathbf{p}_{i}$ is the natural decomposition of $\mathbf{u}$. Then, it follows from Lemma 4.1 that $\mathbf{u}^{\prime}$ can be decomposed as $\mathbf{u}^{\prime}=\prod_{i=1}^{m} \mathbf{p}_{i}^{\prime}$ with $\mathbf{p}_{i} \approx \mathbf{p}_{i}^{\prime} \in \operatorname{id}\left(\mathbf{L} \times \mathbf{Z}_{n}^{\mathbb{R}}\right)$ for all $i$.

Case 1: The word $\mathbf{p}_{i}$ is a singleton. Since $\mathbf{p}_{i} \approx \mathbf{p}_{i}^{\prime} \in \mathrm{id}(\mathbf{L})$, it follows from Lemma 2.1 that $\mathbf{p}_{i}, \mathbf{p}_{i}^{\prime} \in\left\{x, x^{*}\right\}$ for some $x \in \mathscr{A}$. But $\mathbf{p}_{i} \approx \mathbf{p}_{i}^{\prime} \in \operatorname{id}\left(\mathbf{Z}_{n}^{\mathbb{R}}\right)$ and $x \approx x^{*} \notin \operatorname{id}\left(\mathbf{Z}_{n}^{\mathbb{R}}\right)$ imply that $\mathbf{p}_{i}=\mathbf{p}_{i}^{\prime}$.

Case 2: The word $\mathbf{p}_{i}$ is connected. Then, $\mathbf{p}_{i}^{\prime}$ is connected by Lemma 4.1.

Therefore, for each $i$, the identity $\mathbf{p}_{i} \approx \mathbf{p}_{i}^{\prime}$ is either trivial or connected. Consequently, when the identity $\mathbf{u} \approx \mathbf{u}^{\prime}$ in $\Sigma$ is replaced by the connected identities from $\left\{\mathbf{p}_{i} \approx \mathbf{p}_{i}^{\prime}: 1 \leq i \leq m\right\}$, the resulting set remains a basis for $\mathbf{L} \times \mathbf{Z}_{n}^{\mathbb{R}}$.

\subsection{Sandwich identities}

Let $<$ be a total order on the alphabet $\mathscr{A}$. For any nonempty finite subset $\mathscr{X}$ of $\mathscr{A}$, write $\mathscr{X}=\left\{x_{1}<x_{2}<\cdots \prec x_{r}\right\}$ to indicate that $x_{1}, x_{2}, \ldots, x_{r}$ are all the letters of $\mathscr{X}$ with $x_{1}<x_{2}<\cdots<x_{r}$. For such a set $\mathscr{X}$, define

$$
\mathscr{X}^{\boxplus}=\left\{x_{1}^{c_{1}} x_{2}^{c_{2}} \cdots x_{r}^{c_{r}}: c_{1}, c_{2}, \ldots, c_{r} \in\{1,2, \ldots, n\}\right\} .
$$

The shortest word in $\mathscr{X}^{\boxplus}$ is

$$
\overrightarrow{\mathscr{X}}=x_{1} x_{2} \cdots x_{r} .
$$

For any word $\mathbf{w} \in\left(\mathscr{A} \cup \mathscr{A}^{*}\right)^{+}$such that $\operatorname{con}(\overline{\mathbf{w}})=\left\{x_{1} \prec x_{2} \prec \cdots \prec x_{r}\right\}$, define $\min (\mathbf{w})=x_{1}$.

A connected word $s$ is called a sandwich if one of the following holds.

(S.1) $\mathbf{s}=x \mathbf{u} x^{*}$ for some $x \in \mathscr{A}$ and $\mathbf{u} \in\left(\mathscr{A} \cup \mathscr{A}^{*}\right)^{+} \cup\{\varnothing\}$ such that $x \notin \operatorname{con}(\overline{\mathbf{u}})$,

(S.2) $\mathbf{s}=x^{*} \mathbf{u} x$ for some $x \in \mathscr{A}$ and $\mathbf{u} \in\left(\mathscr{A} \cup \mathscr{A}^{*}\right)^{+} \cup\{\varnothing\}$ such that $x \notin \operatorname{con}(\overline{\mathbf{u}})$,

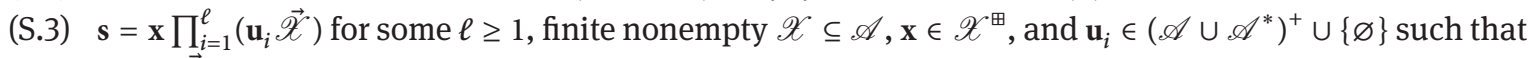

(a) $\overrightarrow{\mathscr{X}}, \mathbf{u}_{1}, \mathbf{u}_{2}, \ldots, \mathbf{u}_{\ell}$ are pairwise disjoint and

(b) if $\ell \geq 2$, then $\mathbf{u}_{i} \neq \varnothing$ for all $i$ and $\min \left(\mathbf{u}_{1}\right)<\min \left(\mathbf{u}_{2}\right)<\cdots<\min \left(\mathbf{u}_{\ell}\right)$.

Specifically, for any $k \in\{1,2,3\}$, a sandwich from (S.k) is said to be of type (S.k). The level of the sandwich in (S.3) is the number $\ell$, while the level of any sandwich in (S.1) and (S.2) is defined to be one.

Remark 4.3. In (S.3), due to (b), the only case in which any of $\mathbf{u}_{1}, \mathbf{u}_{2}, \ldots, \mathbf{u}_{\ell}$ can be empty is when $\ell=1$ and $\mathbf{u}_{1}=\varnothing$, that is, $\mathbf{s}=\mathbf{x} \overrightarrow{\mathscr{X}}$.

An identity $\mathbf{s} \approx \mathbf{s}^{\prime}$ is a sandwich identity if the words $s$ and $\mathbf{s}^{\prime}$ are sandwiches. Denote by

$$
\operatorname{id}_{\mathrm{SAN}}\left(\mathbf{L} \times \mathbf{Z}_{n}^{\mathbb{R}}\right)
$$

the set of all sandwich identities satisfied by the involution semigroup $\mathbf{L} \times \mathbf{Z}_{n}^{\mathbb{R}}$.

Lemma 4.4. The set $\{(3.1)\} \cup \mathrm{id}_{\mathrm{SAN}}\left(\mathbf{L} \times \mathbf{Z}_{n}^{\mathbb{R}}\right)$ constitutes a basis for the involution semigroup $\mathbf{L} \times \mathbf{Z}_{n}^{\mathbb{R}}$.

Recall from Lemma 4.2 that the set id $C\left(\mathbf{L} \times \mathbf{Z}_{n}^{\mathbb{R}}\right)$ of all connected identities from id $\left(\mathbf{L} \times \mathbf{Z}_{n}^{\mathbb{R}}\right)$ constitutes a basis for $\mathbf{L} \times \mathbf{Z}_{n}^{\mathbb{R}}$. As shown in Lemma 3.2, the involution semigroup $\mathbf{L} \times \mathbf{Z}_{n}^{\mathbb{R}}$ satisfies identities (3.1), so that the set $\{(3.1)\} \cup \operatorname{id}_{C}\left(\mathbf{L} \times \mathbf{Z}_{n}^{\mathbb{R}}\right)$ is also a basis for $\mathbf{L} \times \mathbf{Z}_{n}^{\mathbb{R}}$. It is shown in Lemma 4.7 that identities (3.1) can be used to convert any connected word into a sandwich. Lemma 4.4 thus follows.

Lemma 4.5 ([10, Lemma 4.5]). The following identities are deducible from (3.1):

$$
\begin{aligned}
\left(\prod_{i=1}^{r} x_{i}^{c_{i}}\right) \mathbf{h}\left(\prod_{i=1}^{r} x_{i}\right)^{2} \approx\left(\prod_{i=1}^{r} x_{i}^{c_{i}+1}\right) \mathbf{h}\left(\prod_{i=1}^{r} x_{i}\right), \quad c_{i} \geq 1, r \geq 1, \\
\left(\prod_{i=1}^{r} x_{i}^{c_{i}}\right)\left(\prod_{i=1}^{r} x_{i}\right) \mathbf{h}\left(\prod_{i=1}^{r} x_{i}\right) \approx\left(\prod_{i=1}^{r} x_{i}^{c_{i}+1}\right) \mathbf{h}\left(\prod_{i=1}^{r} x_{i}\right), \quad c_{i} \geq 1, r \geq 1,
\end{aligned}
$$

where $\mathbf{h} \in\{\varnothing, h\}$. 
Lemma 4.6. Suppose that $\mathbf{w}$ is any connected word. Then, identities (3.1) can be used to convert $\mathbf{w}$ into some word $\mathbf{w}^{\prime}$ such that $\triangleright \overline{\mathbf{w}^{\prime}}=\overline{\mathbf{w}^{\prime}} \triangleleft$.

Proof. Suppose that $\triangleright \overline{\mathbf{w}} \neq \overline{\mathbf{w}} \triangleleft$. Then, since the word $\mathbf{w}$ is connected, there exist distinct letters $x_{1}, \ldots, x_{r} \in \mathscr{A}$ occurring in $\mathbf{w}$ in the overlapping manner

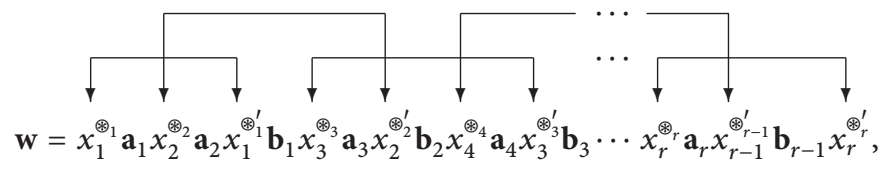

where $\mathbf{a}_{i}, \mathbf{b}_{i} \in\left(\mathscr{A} \cup \mathscr{A}^{*}\right)^{+} \cup\{\varnothing\}$ and $\circledast_{i}, \circledast_{i}^{\prime} \in\{1, *\}$. (Note that $\mathbf{a}_{i}$ follows $x_{i}^{\circledast_{i}}$ while $\mathbf{b}_{i}$ follows $x_{i}^{\circledast_{i}^{\prime}}$.) Then, identities $\{(3.1 \mathrm{~b}),(3.1 \mathrm{i}),(3.1 \mathrm{j}),(3.1 \mathrm{k}),(3.11)\}$ can be used to convert $\mathbf{w}$ into

$$
x_{1}^{p_{1}} \mathbf{a}_{1} x_{2}^{p_{2}} \mathbf{a}_{2} x_{1} \mathbf{b}_{1} x_{3}^{p_{3}} \mathbf{a}_{3} x_{2} \mathbf{b}_{2} x_{4}^{p_{4}} \mathbf{a}_{4} x_{3} \mathbf{b}_{3} \cdots x_{r}^{p_{r}} \mathbf{a}_{r} x_{r-1} \mathbf{b}_{r-1} x_{r},
$$

where $p_{i}, q_{i} \geq 1$. Hence,

$$
\begin{aligned}
& \mathbf{w} \underset{\stackrel{(3.1)}{\approx}}{\sim} x_{1}^{p_{1}} \mathbf{a}_{1} x_{2}^{p_{2}} \mathbf{a}_{2} x_{1} \mathbf{b}_{1} x_{3}^{p_{3}} \mathbf{a}_{3} x_{2} \mathbf{b}_{2} x_{4}^{p_{4}} \mathbf{a}_{4} x_{3} \mathbf{b}_{3} \cdots x_{r}^{p_{r}} \mathbf{a}_{r} x_{r-1} \mathbf{b}_{r-1} x_{r} \\
& \stackrel{(3.1 v)}{\approx} x_{1}^{p_{1}} \mathbf{a}_{1} x_{2}^{p_{2}} \mathbf{a}_{2} x_{1} \mathbf{b}_{1} x_{3}^{p_{3}} \mathbf{a}_{3} x_{2} x_{1}^{n} \mathbf{b}_{2} x_{4}^{p_{4}} \mathbf{a}_{4} x_{3} \mathbf{b}_{3} \cdots x_{r}^{p_{r}} \mathbf{a}_{r} x_{r-1} \mathbf{b}_{r-1} x_{r} \\
& \stackrel{(3.1 v)}{\sim} x_{1}^{p_{1}} \mathbf{a}_{1} x_{2}^{p_{2}} \mathbf{a}_{2} x_{1} \mathbf{b}_{1} x_{3}^{p_{3}} \mathbf{a}_{3} x_{2} x_{1}^{n} \mathbf{b}_{2} x_{4}^{p_{4}} \mathbf{a}_{4} x_{3} x_{1}^{n} \mathbf{b}_{3} \cdots x_{r}^{p_{r}} \mathbf{a}_{r} x_{r-1} \mathbf{b}_{r-1} x_{r} \\
& \quad \vdots \\
& \stackrel{(3.1 v)}{\approx} x_{1}^{p_{1}} \mathbf{a}_{1} x_{2}^{p_{2}} \mathbf{a}_{2} x_{1} \mathbf{b}_{1} x_{3}^{p_{3}} \mathbf{a}_{3} x_{2} x_{1}^{n} \mathbf{b}_{2} x_{4}^{p_{4}} \mathbf{a}_{4} x_{3} x_{1}^{n} \mathbf{b}_{3} \cdots x_{r}^{p_{r}} \mathbf{a}_{r} x_{r-1} x_{1}^{n} \mathbf{b}_{r-1} x_{r} \\
& \stackrel{(3.1 v)}{\approx} x_{1}^{p_{1}} \mathbf{a}_{1} x_{2}^{p_{2}} \mathbf{a}_{2} x_{1} \mathbf{b}_{1} x_{3}^{p_{3}} \mathbf{a}_{3} x_{2} x_{1}^{n} \mathbf{b}_{2} x_{4}^{p_{4}} \mathbf{a}_{4} x_{3} x_{1}^{n} \mathbf{b}_{3} \cdots x_{r}^{p_{r}} \mathbf{a}_{r} x_{r-1} x_{1}^{n} \mathbf{b}_{r-1} x_{r} x_{1}^{n} \\
& =\mathbf{w}^{\prime},
\end{aligned}
$$

where $\triangleright \overline{\mathbf{w}^{\prime}}=x_{1}=\overline{\mathbf{w}^{\prime}} \triangleleft$.

Lemma 4.7. Suppose that $\mathbf{w}$ is any connected word. Then, identities (3.1) can be used to convert $\mathbf{w}$ into some sandwich $\mathbf{s}$ with $\operatorname{sim}(\overline{\mathbf{w}})=\operatorname{sim}(\overline{\mathbf{s}})$ and $\operatorname{non}(\overline{\mathbf{w}})=\operatorname{non}(\overline{\mathbf{s}})$.

Proof. By Lemma 4.5, it suffices to convert the word $\mathbf{w}$, using identities (3.1) and (4.1), into some sandwich $\mathbf{s}$ with $\operatorname{sim}(\overline{\mathbf{w}})=\operatorname{sim}(\overline{\mathbf{s}})$ and $\operatorname{non}(\overline{\mathbf{w}})=\operatorname{non}(\overline{\mathbf{s}})$. By Lemma 4.6, it can be assumed that $x_{1}=\triangleright \overline{\mathbf{w}}=\overline{\mathbf{w}} \triangleleft$. Then, $\mathbf{w}$ can be written as

$$
\mathbf{w}=x_{1}^{\circledast_{0}} \prod_{i=1}^{m_{1}}\left(\mathbf{w}_{1, i} x_{1}^{\oplus_{i}}\right),
$$

where $m_{1} \geq 1, \circledast_{i} \in\{1, *\}$, and $\mathbf{w}_{1, i} \in\left(\mathscr{A} \cup \mathscr{A}^{*}\right)^{+} \cup\{\varnothing\}$ with $x_{1} \notin \operatorname{con}\left(\overline{\mathbf{w}_{1, i}}\right)$ for all $i$. If $m_{1}=1$, then $\mathbf{w}$ is either $x_{1} \mathbf{w}_{1,1} x_{1}, x_{1} \mathbf{w}_{1,1} x_{1}^{*}, x_{1}^{*} \mathbf{w}_{1,1} x_{1}$, or $x_{1}^{*} \mathbf{w}_{1,1} x_{1}^{*}$; the first three words are sandwiches while identities (3.1) can be used to convert the fourth word into a sandwich of type (S.3), that is,

$$
x_{1}^{*} \mathbf{w}_{1,1} x_{1}^{*} \stackrel{(3.1 \mathrm{e})}{\approx} x_{1}^{\mathbb{R}} \mathbf{w}_{1,1} x_{1}^{\mathbb{R}} \stackrel{(3.1 \mathrm{lb})}{\approx} x_{1}^{2 \mathbb{R}-1} \mathbf{w}_{1,1} x_{1} \stackrel{(3.1 \mathrm{a})}{\approx} x_{1}^{c} \mathbf{w}_{1,1} x_{1},
$$

where $c \in\{1,2, \ldots, n\}$ is such that $c \equiv 2 \mathbb{R}-1(\bmod n)$. Therefore, assume that $m_{1} \geq 2$, so that identities (3.1e)(3.1h) can be used to replace any $x_{1}^{*}$ by $x_{1}^{\mathbb{R}}$.

Suppose that $\mathbf{w}_{1, i}$ and $\mathbf{w}_{1, j}$ are not disjoint with $i \neq j$, say $x_{2} \in \operatorname{con}\left(\overline{\mathbf{w}_{1, i}}\right) \cap \operatorname{con}\left(\overline{\mathbf{w}_{1, j}}\right)$. Then,

$$
\mathbf{w}=x_{1} \mathbf{h}_{1} x_{2}^{\otimes_{1}^{\prime}} \mathbf{h}_{2} x_{1} \mathbf{h}_{3} x_{2}^{\otimes_{2}^{\prime}} \mathbf{h}_{4} x_{1}
$$

for some $\mathbf{h}_{i} \in\left(\mathscr{A} \cup \mathscr{A}^{*}\right)^{+} \cup\{\varnothing\}$ and $\circledast_{i}^{\prime} \in\{1, *\}$. Identities (3.1i)-(3.11) can first be used to replace any $x_{2}^{*}$ by $x_{2}^{\mathbb{R}}$ and identities (3.10)-(3.1v) can then be used to perform the replacement $\left(x_{1}, x_{2}\right) \mapsto\left(x_{1} x_{2}^{n}, x_{1}^{n} x_{2}\right)$. The resulting word is of the form

$$
x_{1}^{c_{0,1}} x_{2}^{c_{0,2}} \prod_{i=1}^{m_{2}}\left(\mathbf{w}_{2, i} x_{1}^{c_{i, 1}} x_{2}^{c_{i, 2}}\right)
$$


where $m_{2} \geq 2, c_{i, j} \geq 1$, and $\mathbf{w}_{2, i} \in\left(\mathscr{A} \cup \mathscr{A}^{*}\right)^{+} \cup\{\varnothing\}$ with $x_{1}, x_{2} \notin \operatorname{con}\left(\overline{\mathbf{w}_{2, i}}\right)$ for all $i$. Similarly, if $\mathbf{w}_{2, i}$ and $\mathbf{w}_{2, j}$ are not disjoint with $i \neq j$, say $x_{3} \in \operatorname{con}\left(\overline{\mathbf{w}_{2, i}}\right) \cap \operatorname{con}\left(\overline{\mathbf{w}_{2, j}}\right)$, then identities (3.1i)-(3.11) can first be used to replace any $x_{3}^{*}$ by $x_{3}^{\mathbb{R}}$ and identities (3.10)-(3.1v) can then be used to perform the replacement $\left(x_{2}^{c_{i, 2}}, x_{3}\right) \mapsto\left(x_{2}^{c_{i, 2}} x_{3}^{n}, x_{1}^{n} x_{2}^{n} x_{3}\right)$. The resulting word is of the form

$$
x_{1}^{c_{0,1}} x_{2}^{c_{0,2}} x_{3}^{c_{0,3}} \prod_{i=1}^{m_{3}}\left(\mathbf{w}_{3, i} x_{1}^{c_{i, 1}} x_{2}^{c_{i, 2}} x_{3}^{c_{i, 3}}\right)
$$

where $m_{3} \geq 2, c_{i, j} \geq 1$, and $\mathbf{w}_{3, i} \in\left(\mathscr{A} \cup \mathscr{A}^{*}\right)^{+} \cup\{\varnothing\}$ with $x_{1}, x_{2}, x_{3} \notin \operatorname{con}\left(\overline{\mathbf{w}_{3, i}}\right)$ for all $i$. This can be repeated until a word of the form

$$
x_{1}^{c_{0,1}} x_{2}^{c_{0,2}} \cdots x_{r}^{c_{0, r}} \prod_{i=1}^{m_{r}}\left(\mathbf{w}_{r, i} x_{1}^{c_{i, 1}} x_{2}^{c_{i, 2}} \cdots x_{r}^{c_{i, r}}\right)
$$

is obtained, where $m_{r} \geq 2, c_{i, j} \geq 1$, and $\mathbf{w}_{r, 1}, \mathbf{w}_{r, 2}, \ldots, \mathbf{w}_{r, m_{r}} \in\left(\mathscr{A} \cup \mathscr{A}^{*}\right)^{+} \cup\{\varnothing\}$ are pairwise disjoint words with $x_{1}, x_{2}, \ldots, x_{r} \notin \operatorname{con}\left(\overline{\mathbf{w}_{r, i}}\right)$ for all $i$. It is easily seen that identities (3.1a) and (3.1b) can be used to convert this word into

$$
\mathbf{w}^{\prime}=x_{1}^{c_{1}} x_{2}^{c_{2}} \cdots x_{r}^{c_{r}} \prod_{i=1}^{m_{r}}\left(\mathbf{w}_{r, i} x_{1} x_{2} \cdots x_{r}\right),
$$

where $1 \leq c_{i} \leq n$. Let $\pi$ denote the permutation on $\{1,2, \ldots, r\}$ such that

$$
x_{1 \pi}<x_{2 \pi}<\cdots<x_{r \pi} .
$$

Then,

$$
\begin{aligned}
& \mathbf{w}^{\prime} \stackrel{(3.1 \mathrm{a})}{\approx} x_{1}^{c_{1}} x_{2}^{c_{2}} \cdots x_{r}^{c_{r}} \mathbf{w}_{r, 1} x_{1} x_{2} \cdots x_{r} \prod_{i=2}^{m_{r}}\left(\mathbf{w}_{r, i}\left(x_{1} x_{2} \cdots x_{r}\right)^{n+1}\right) \\
& \stackrel{(3.1 \mathrm{n})}{\approx} x_{1 \pi}^{c_{1 \pi} \pi} x_{2 \pi}^{c_{2 \pi}} \cdots x_{r \pi}^{c_{r \pi}} \mathbf{w}_{r, 1} x_{1 \pi} x_{2 \pi} \cdots x_{r \pi} \prod_{i=2}^{m_{r}}\left(\mathbf{w}_{r, i}\left(x_{1} x_{2} \cdots x_{r}\right)^{n+1}\right) \\
& \quad \stackrel{(3.1 \mathrm{~m})}{\approx} x_{1 \pi}^{c_{1 \pi}} x_{2 \pi}^{c_{2 \pi}} \cdots x_{r \pi}^{c_{r \pi}} \mathbf{w}_{r, 1} x_{1 \pi} x_{2 \pi} \cdots x_{r \pi} \prod_{i=2}^{m_{r}}\left(\mathbf{w}_{r, i}\left(x_{1 \pi} x_{2 \pi} \cdots x_{r \pi}\right)^{n+1}\right) \\
& \stackrel{(3.1 \mathrm{a})}{\approx} x_{1 \pi}^{c_{1 \pi}} x_{2 \pi}^{c_{2 \pi}} \cdots x_{r \pi}^{c_{r \pi}} \prod_{i=1}^{m_{r}}\left(\mathbf{w}_{r, i} x_{1 \pi} x_{2 \pi} \cdots x_{r \pi}\right) .
\end{aligned}
$$

In summary, identities (3.1) can be used to convert w into a word of the form

$$
\mathbf{s}=\mathbf{x} \prod_{i=1}^{\ell}\left(\mathbf{u}_{i} \overrightarrow{\mathscr{X}}\right)
$$

where $\ell \geq 1, \mathscr{X}=\left\{x_{1} \prec x_{2} \prec \cdots \prec x_{r}\right\} \subseteq \mathscr{A}, \mathbf{x}=x_{1}^{c_{1}} x_{2}^{c_{2}} \cdots x_{r}^{c_{r}} \in \mathscr{X}^{\boxplus}$, and $\mathbf{u}_{i} \in\left(\mathscr{A} \cup \mathscr{A}^{*}\right)^{+} \cup\{\varnothing\}$ are such that $\overrightarrow{\mathscr{X}}, \mathbf{u}_{1}, \mathbf{u}_{2}, \ldots, \mathbf{u}_{\ell}$ are pairwise disjoint. Let $c_{i}^{\prime}$ be the number in $\{1,2, \ldots, n\}$ such that $c_{i}+1 \equiv c_{i}^{\prime}(\bmod n)$. If $\mathbf{u}_{1}=\varnothing$, then

$$
\mathbf{s}=x_{1}^{c_{1}} x_{2}^{c_{2}} \cdots x_{r}^{c_{r}} \overrightarrow{\mathscr{X}} \mathbf{u}_{2} \overrightarrow{\mathscr{X}} \prod_{i=3}^{\ell}\left(\mathbf{u}_{i} \overrightarrow{\mathscr{X}}\right) \stackrel{(4.1 \mathrm{~b})}{\sim} x_{1}^{c_{1}+1} x_{2}^{c_{2}+1} \cdots x_{r}^{c_{r}+1} \mathbf{u}_{2} \overrightarrow{\mathscr{X}} \prod_{i=3}^{\ell}\left(\mathbf{u}_{i} \overrightarrow{\mathscr{X}}\right) \stackrel{(3.1 \mathrm{a})}{\approx} x_{1}^{c_{1}^{\prime}} x_{2}^{c_{2}^{\prime}} \cdots x_{r}^{c_{r}^{\prime}} \mathbf{u}_{2} \overrightarrow{\mathscr{X}} \prod_{i=3}^{\ell}\left(\mathbf{u}_{i} \overrightarrow{\mathscr{X}}\right)
$$

and if $\mathbf{u}_{j}=\varnothing$ for some $j \in\{2,3, \ldots, \ell\}$, then

$$
\begin{aligned}
& \mathbf{s}=x_{1}^{c_{1}} x_{2}^{c_{2}} \cdots x_{r}^{c_{r}}\left(\prod_{i=1}^{j-1}\left(\mathbf{u}_{i} \overrightarrow{\mathscr{X}}\right)\right) \overrightarrow{\mathscr{X}}\left(\prod_{i=j+1}^{\ell}\left(\mathbf{u}_{i} \overrightarrow{\mathscr{X}}\right)\right) \\
& \stackrel{(4.1)}{\approx} x_{1}^{c_{1}+1} x_{2}^{c_{2}+1} \cdots x_{r}^{c_{r}+1}\left(\prod_{i=1}^{j-1}\left(\mathbf{u}_{i} \overrightarrow{\mathscr{X}}\right)\right)\left(\prod_{i=j+1}^{\ell}\left(\mathbf{u}_{i} \overrightarrow{\mathscr{X}}\right)\right) \\
& \stackrel{(3.1 \mathrm{a})}{\approx} x_{1}^{c_{1}^{\prime}} x_{2}^{c_{2}^{\prime}} \cdots x_{r}^{c_{r}^{\prime}}\left(\prod_{i=1}^{j-1}\left(\mathbf{u}_{i} \overrightarrow{\mathscr{X}}\right)\right)\left(\prod_{i=j+1}^{\ell}\left(\mathbf{u}_{i} \overrightarrow{\mathscr{X}}\right)\right) .
\end{aligned}
$$

Hence, for any $i$, if the factor $\mathbf{u}_{i}$ is empty, then the $\overrightarrow{\mathscr{X}}$ that follows it can be "combined" with the prefix $\mathbf{x}$. Therefore, it can further be assumed that either

(A) $\mathbf{s}=\mathbf{x} \overrightarrow{\mathscr{X}}$ or

(B) $\mathbf{u}_{i} \neq \varnothing$ for all $i$. 
If (A) holds, then the word $s$ is a sandwich of type (S.3). Hence, assume that (B) holds. It remains to show that if $\ell \geq 2$, then identities (3.1) can be used to rearrange the factors $\mathbf{u}_{1}, \mathbf{u}_{2}, \ldots, \mathbf{u}_{\ell}$, so that $\mathbf{s}$ satisfies (S.3)(b) and is a sandwich of type (S.3). To interchange $\mathbf{u}_{j}$ and $\mathbf{u}_{j+1}$ for any $j \geq 2$, identity (3.1c) can clearly be used. To interchange $\mathbf{u}_{1}$ and $\mathbf{u}_{2}$,

$$
\begin{aligned}
& \mathbf{s} \stackrel{(3.1 \mathrm{aa})}{\approx} \mathbf{x u}_{1} \overrightarrow{\mathscr{X}} \mathbf{u}_{2} \overrightarrow{\mathscr{X}} \overrightarrow{\mathscr{X}} \overrightarrow{\mathscr{X}}^{n-1} \prod_{i=3}^{\ell}\left(\mathbf{u}_{i} \overrightarrow{\mathscr{X}}\right) \\
& \stackrel{(3.1 \mathrm{~b})}{\approx} \overrightarrow{\mathscr{X}} \mathbf{u}_{1} \overrightarrow{\mathscr{X}} \mathbf{u}_{2} \overrightarrow{\mathscr{X}} \mathbf{x} \overrightarrow{\mathscr{X}}^{n-1} \prod_{i=3}^{\ell}\left(\mathbf{u}_{i} \overrightarrow{\mathscr{X}}\right) \\
& \stackrel{(3.1 \mathrm{c})}{\approx} \overrightarrow{\mathscr{X}} \mathbf{u}_{2} \overrightarrow{\mathscr{X}} \mathbf{u}_{1} \overrightarrow{\mathscr{X}} \mathbf{x} \overrightarrow{\mathscr{X}}^{n-1} \prod_{i=3}^{\ell}\left(\mathbf{u}_{i} \overrightarrow{\mathscr{X}}\right) \\
& \stackrel{(3.1 \mathrm{~b})}{\approx} \mathbf{x u}_{2} \overrightarrow{\mathscr{X}} \mathbf{u}_{1} \overrightarrow{\mathscr{X}} \overrightarrow{\mathscr{X}} \overrightarrow{\mathscr{X}}^{n-1} \prod_{i=3}^{\ell}\left(\mathbf{u}_{i} \overrightarrow{\mathscr{X}}\right) \\
& \stackrel{(3.1 \mathrm{aa})}{\approx} \mathbf{x u}_{2} \overrightarrow{\mathscr{X}} \mathbf{u}_{1} \overrightarrow{\mathscr{X}} \prod_{i=3}^{\ell}\left(\mathbf{u}_{i} \overrightarrow{\mathscr{X}}\right) .
\end{aligned}
$$

Throughout this proof, identities (3.1) have been used to convert the word $w$ into a sandwich $\mathbf{s}$. Therefore, $\mathbf{w} \approx \mathbf{s} \in \operatorname{id}(\mathbf{L})$ by Lemma 3.2, so that $\operatorname{sim}(\overline{\mathbf{w}})=\operatorname{sim}(\overline{\mathbf{s}})$ and $\operatorname{non}(\overline{\mathbf{w}})=\operatorname{non}(\overline{\mathbf{s}})$ by Lemma 2.1.

\section{Restrictions on sandwich identities}

The present section establishes some properties of sandwich identities satisfied by the involution semigroup $\mathbf{L} \times \mathbf{Z}_{n}^{\mathbb{R}}$. In Section 5.1, it is shown that any two sandwiches that form such an identity must share the same type and level. In Section 5.2, refined identities are introduced; these are identities formed by certain sandwiches of level one. It is shown in Lemma 5.8 that refined identities satisfied by the involution semigroup $\mathbf{L} \times \mathbf{Z}_{n}^{\mathbb{R}}$ are of very specific form.

The results established in the present section are required in Section 6 in the proof of Theorem 3.1.

\subsection{Type of sandwiches forming sandwich identities}

Lemma 5.1. Suppose that $\mathbf{s} \approx \mathbf{s}^{\prime} \in \mathrm{id}_{\mathrm{SAN}}\left(\mathbf{L} \times \mathbf{Z}_{n}^{\mathbb{R}}\right)$, where $\mathbf{s}=x \mathbf{u} x^{*}$ is the sandwich in (S.1). Then, $\mathbf{s}^{\prime}=x \mathbf{u}^{\prime} x^{*}$ for some $\mathbf{u}^{\prime} \in\left(\mathscr{A} \cup \mathscr{A}^{*}\right)^{+} \cup\{\varnothing\}$ with $x \notin \operatorname{con}(\overline{\mathbf{u}})=\operatorname{con}\left(\overline{\mathbf{u}^{\prime}}\right)$. Consequently, s is of type (S.1) if and only if $\mathbf{s}^{\prime}$ is of type (S.1).

Proof. Let $\varphi: \mathscr{A} \rightarrow L$ denote the substitution given by

$$
z \varphi= \begin{cases}f e & \text { if } z=x \\ e & \text { otherwise }\end{cases}
$$

Then, $\mathbf{s} \varphi=f e f=\mathbf{s}^{\prime} \varphi$. It follows that

$$
\mathbf{s}^{\prime} \varphi=f e \cdot e \cdot e \cdots e \cdot e f,
$$

whence $\mathbf{s}^{\prime}=x \mathbf{u}^{\prime} x^{*}$ for some $\mathbf{u}^{\prime} \in\left(\mathscr{A} \cup \mathscr{A}^{*}\right)^{+} \cup\{\varnothing\}$ with $x \notin \operatorname{con}\left(\overline{\mathbf{u}^{\prime}}\right)$. Furthermore, con $(\overline{\mathbf{s}})=\operatorname{con}\left(\overline{\mathbf{s}^{\prime}}\right)$ by Lemma 2.1, so that $x \notin \operatorname{con}(\overline{\mathbf{u}})=\operatorname{con}\left(\overline{\mathbf{u}^{\prime}}\right)$.

Lemma 5.2. Suppose that $\mathbf{s} \approx \mathbf{s}^{\prime} \in \mathrm{id}_{\mathrm{SAN}}\left(\mathbf{L} \times \mathbf{Z}_{n}^{\mathbb{R}}\right)$, where $\mathbf{s}=x^{*} \mathbf{u} x$ is the sandwich in (S.2). Then, $\mathbf{s}^{\prime}=x^{*} \mathbf{u}^{\prime} x$ for some $\mathbf{u}^{\prime} \in\left(\mathscr{A} \cup \mathscr{A}^{*}\right)^{+} \cup\{\varnothing\}$ with $x \notin \operatorname{con}(\overline{\mathbf{u}})=\operatorname{con}\left(\overline{\mathbf{u}^{\prime}}\right)$. Consequently, s is of type (S.2) if and only if $\mathbf{s}^{\prime}$ is of type (S.2).

Proof. This is symmetric to Lemma 5.1. 
Lemma 5.3. Suppose that $\mathbf{s} \approx \mathbf{s}^{\prime} \in \mathrm{id}_{\mathrm{SAN}}\left(\mathbf{L} \times \mathbf{Z}_{n}^{\mathbb{R}}\right)$, where

$$
\mathbf{s}=\mathbf{x} \prod_{i=1}^{\ell}\left(\mathbf{u}_{i} \overrightarrow{\mathscr{X}}\right)
$$

is the sandwich in (S.3). Then,

$$
\mathbf{s}^{\prime}=\mathbf{x} \prod_{i=1}^{\ell}\left(\mathbf{u}_{i}^{\prime} \overrightarrow{\mathscr{X}}\right)
$$

for some $\mathbf{u}_{1}^{\prime}, \mathbf{u}_{2}^{\prime}, \ldots, \mathbf{u}_{\ell}^{\prime} \in\left(\mathscr{A} \cup \mathscr{A}^{*}\right)^{+} \cup\{\varnothing\}$ such that $\operatorname{con}\left(\overline{\mathbf{u}_{i}}\right)=$ con $\left(\overline{\mathbf{u}_{i}^{\prime}}\right)$ for all $i$. Consequently, s is of type (S.3) if and only if $\mathbf{s}^{\prime}$ is of type (S.3).

Proof. Following the proof of [10, Lemma 5.3], there holds

$$
\mathbf{s}^{\prime}=\mathbf{y} \prod_{i=1}^{\ell}\left(\mathbf{u}_{i}^{\prime} \overrightarrow{\mathscr{X}}\right)
$$

for some $\mathbf{y} \in \mathscr{X}^{\boxplus}$ and $\mathbf{u}_{1}^{\prime}, \mathbf{u}_{2}^{\prime}, \ldots, \mathbf{u}_{\ell}^{\prime} \in\left(\mathscr{A} \cup \mathscr{A}^{*}\right)^{+} \cup\{\varnothing\}$ such that $\operatorname{con}\left(\overline{\mathbf{u}_{i}}\right)=\operatorname{con}\left(\overline{\mathbf{u}_{i}^{\prime}}\right)$ for all $i$. Since

$$
\mathscr{X} \cap \operatorname{con}\left(\overline{\mathbf{u}_{1}} \cdot \overline{\mathbf{u}_{2}} \cdots \overline{\mathbf{u}_{\ell}}\right)=\varnothing
$$

by (S.3)(a) and $\mathbf{s} \approx \mathbf{s}^{\prime} \in \mathrm{id}\left(\mathbf{Z}_{n}^{\mathbb{R}}\right)$, where $\mathbb{Z}_{n}$ has a unit element, $\mathbf{Z}_{n}^{\mathbb{R}}$ satisfies the identity $\mathbf{x} \overrightarrow{\mathscr{X}}^{\ell} \approx \mathbf{y} \overrightarrow{\mathscr{X}}^{\ell}$ obtained from $\mathbf{s} \approx \mathbf{s}^{\prime}$ by retaining the letters in $\mathscr{X}$. Since $\mathbf{x} \overrightarrow{\mathscr{X}}^{\ell}, \mathbf{y} \overrightarrow{\mathscr{X}}^{\ell} \in \mathscr{A}^{+}$, it follows from Lemma 2.2 that $\mathbf{x}=\mathbf{y}$.

Lemma 5.4. Suppose that $\mathbf{s} \approx \mathbf{s}^{\prime} \in \mathrm{id}_{\mathrm{SAN}}\left(\mathbf{L} \times \mathbf{Z}_{n}^{\mathbb{R}}\right)$, where

$$
\mathbf{s}=\mathbf{x} \prod_{i=1}^{\ell}\left(\mathbf{u}_{i} \overrightarrow{\mathscr{X}}\right) \quad \text { and } \quad \mathbf{s}^{\prime}=\mathbf{x} \prod_{i=1}^{\ell}\left(\mathbf{u}_{i}^{\prime} \overrightarrow{\mathscr{X}}\right)
$$

are the sandwiches of type (S.3) in Lemma 5.3. Then,

$$
\left\{(3.1), \mathbf{s} \approx \mathbf{s}^{\prime}\right\} \sim\{(3.1)\} \cup\left\{x \mathbf{u}_{i} x \approx x \mathbf{u}_{i}^{\prime} x: 1 \leq i \leq \ell\right\} .
$$

Proof. For each $i \in\{1,2, \ldots, \ell\}$, let $\varphi_{i}: \mathscr{A} \rightarrow \mathscr{A}^{+}$denote the substitution given by

$$
z \varphi_{i}= \begin{cases}z & \text { if } z \in \operatorname{con}\left(\overline{\mathbf{u}_{i}}\right)=\operatorname{con}\left(\overline{\mathbf{u}_{i}^{\prime}}\right), \\ x^{n} & \text { otherwise. }\end{cases}
$$

Note that for any $\mathbf{w} \in\left(\mathscr{A} \cup \mathscr{A}^{*}\right)^{+}$such that $\mathbf{w}$ and $\mathbf{u}_{i}$ are disjoint, the image $\mathbf{w} \varphi_{i}$ belongs to $\left\{x^{n},\left(x^{n}\right)^{*}\right\}^{+}$. Therefore, identity (3.1d) can be used to convert $\mathbf{w} \varphi_{i}$ into the plain word $\overline{\mathbf{w} \varphi_{i}}$ in $\left\{x^{n}\right\}^{+}$. Hence, by (S.3)(a),

$$
\begin{aligned}
x\left(\mathbf{s} \varphi_{i}\right) x & =x\left(\left(\mathbf{x} \mathbf{u}_{1} \overrightarrow{\mathscr{X}} \cdots \mathbf{u}_{i-1} \overrightarrow{\mathscr{X}}\right) \varphi_{i}\right) \mathbf{u}_{i}\left(\left(\overrightarrow{\mathscr{X}} \mathbf{u}_{i+1} \overrightarrow{\mathscr{X}} \cdots \mathbf{u}_{\ell} \overrightarrow{\mathscr{X}}\right) \varphi_{i}\right) x \\
& \stackrel{(3.1 \mathrm{~d})}{\approx} x\left(\overline{\left(\mathbf{x} \mathbf{u}_{1} \overrightarrow{\mathscr{X}} \cdots \mathbf{u}_{i-1} \overrightarrow{\mathscr{X}}\right) \varphi_{i}}\right) \mathbf{u}_{i}\left(\overline{\left(\overrightarrow{\mathscr{X}} \mathbf{u}_{i+1} \overrightarrow{\mathscr{X}} \cdots \mathbf{u}_{\ell} \overrightarrow{\mathscr{X}}\right) \varphi_{i}}\right) x \\
& \stackrel{(3.1 \mathrm{a})}{\approx} x \mathbf{u}_{i} x,
\end{aligned}
$$

so that (3.1) $\vdash x\left(\mathbf{s} \varphi_{i}\right) x \approx x \mathbf{u}_{i} x$. Similarly, the deduction (3.1) $\vdash x\left(\mathbf{s}^{\prime} \varphi_{i}\right) x \approx x \mathbf{u}_{i}^{\prime} x$ holds. Thus, the deduction $\left\{(3.1), \mathbf{s} \approx \mathbf{s}^{\prime}\right\} \vdash x \mathbf{u}_{i} x \approx x \mathbf{u}_{i}^{\prime} x$ holds. Conversely,

$$
\begin{aligned}
\mathbf{s} & =x_{1}^{c_{1}} x_{2}^{c_{2}} \cdots x_{r}^{c_{r}} \mathbf{u}_{1} \overrightarrow{\mathscr{X}} \prod_{i=2}^{\ell}\left(\mathbf{u}_{i} \overrightarrow{\mathscr{X}}\right) \\
& \stackrel{(3.1 \mathrm{t})}{\approx} x_{1}^{c_{1}} x_{2}^{c_{2}} \cdots x_{r}^{c_{r}} \mathbf{u}_{1} x_{r}^{n} \overrightarrow{\mathscr{X}} \prod_{i=2}^{\ell}\left(\mathbf{u}_{i} \overrightarrow{\mathscr{X}}\right) \\
& \approx x_{1}^{c_{1}} x_{2}^{c_{2}} \cdots x_{r}^{c_{r}} \mathbf{u}_{1}^{\prime} x_{r}^{n} \overrightarrow{\mathscr{X}} \prod_{i=2}^{\ell}\left(\mathbf{u}_{i}^{\prime} \overrightarrow{\mathscr{X}}\right) \quad \text { by } x \mathbf{u}_{i} x \approx x \mathbf{u}_{i}^{\prime} x \\
& \stackrel{(3.1 \mathrm{t})}{\approx} \mathbf{s}^{\prime} .
\end{aligned}
$$

Therefore, the deduction $\{(3.1)\} \cup\left\{x \mathbf{u}_{i} x \approx x \mathbf{u}_{i}^{\prime} x: 1 \leq i \leq \ell\right\} \vdash \mathbf{s} \approx \mathbf{s}^{\prime}$ holds. 


\subsection{Refined identities}

By Lemmas 5.1-5.3, any identity in $\operatorname{id}_{\mathrm{SAN}}\left(\mathbf{L} \times \mathbf{Z}_{n}^{\mathbb{R}}\right)$ is formed by a pair of sandwiches that share the same type and level. Therefore, it is unambiguous to define the type and level of a sandwich identity $\mathbf{s} \approx \mathbf{s}^{\prime}$ in $\operatorname{id}_{\text {AN }}\left(\mathbf{L} \times \mathbf{Z}_{n}^{\mathbb{R}}\right)$ to be, respectively, the type and level shared by the sandwiches $\mathbf{s}$ and $\mathbf{s}^{\prime}$. The present subsection investigates identities in $\operatorname{id}_{\mathrm{SAN}}\left(\mathbf{L} \times \mathbf{Z}_{n}^{\mathbb{R}}\right)$ of level one.

Consider a word $\mathbf{r}$ of the form

$$
\mathbf{r}=x\left(\prod_{i=1}^{k} \mathbf{p}_{i}\right) x^{\circledast}
$$

where $k \geq 1, x \in \mathscr{A}, \circledast \in\{1, *\}$, and $\mathbf{p}_{i} \in\left(\mathscr{A} \cup \mathscr{A}^{*}\right)^{+}$are such that $x, \mathbf{p}_{1}, \mathbf{p}_{2}, \ldots, \mathbf{p}_{k}$ are pairwise disjoint. Note that depending on $\circledast$, the word $\mathbf{r}$ is a level one sandwich of type (S.1) or (S.3). This sandwich is said to be refined if it satisfies both that

(R.1) each $\mathbf{p}_{i}$ is either a singleton or a sandwich and

(R.2) if $\mathbf{p}_{1}, \mathbf{p}_{2}, \ldots, \mathbf{p}_{k}$ are all sandwiches with $k \geq 2$, then $\min \left(\mathbf{p}_{1}\right)<\min \left(\mathbf{p}_{k}\right)$.

An identity $\mathbf{r} \approx \mathbf{r}^{\prime}$ is a refined identity if the words $\mathbf{r}$ and $\mathbf{r}^{\prime}$ are refined sandwiches. Denote by

$$
\operatorname{id}_{\mathrm{REF}}\left(\mathbf{L} \times \mathbf{Z}_{n}^{\mathbb{R}}\right)
$$

the set of all refined identities satisfied by the involution semigroup $\mathbf{L} \times \mathbf{Z}_{n}^{\mathbb{R}}$.

Lemma 5.5. Suppose that $\mathbf{s} \approx \mathbf{s}^{\prime} \in \mathrm{id}_{\mathrm{SAN}}\left(\mathbf{L} \times \mathbf{Z}_{n}^{\mathbb{R}}\right)$. Then, there exists some finite subset $\Sigma$ of $\mathrm{id}_{\mathrm{REF}}\left(\mathbf{L} \times \mathbf{Z}_{n}^{\mathbb{R}}\right)$ such that the equivalence $\left\{(3.1), \mathbf{s} \approx \mathbf{s}^{\prime}\right\} \sim\{(3.1)\} \cup \Sigma$ holds. Furthermore, each identity $\mathbf{r} \approx \mathbf{r}^{\prime}$ in $\Sigma$ can be chosen so that $|\operatorname{sim}(\overline{\mathbf{r}})| \leq|\operatorname{sim}(\overline{\mathbf{s}})|$ and $|\operatorname{non}(\overline{\mathbf{r}})| \leq|\operatorname{non}(\overline{\mathbf{s}})|$.

Proof. There are three cases depending on the type of the sandwich identity $\mathbf{s} \approx \mathbf{s}^{\prime}$.

Case 1: The word $s \approx s^{\prime}$ is of type (S.1). Then, by Lemma 5.1,

$$
\mathbf{s}=x \mathbf{u} x^{*} \text { and } \mathbf{s}^{\prime}=x \mathbf{u}^{\prime} x^{*}
$$

for some $x \in \mathscr{A}$ and $\mathbf{u}, \mathbf{u}^{\prime} \in\left(\mathscr{A} \cup \mathscr{A}^{*}\right)^{+} \cup\{\varnothing\}$ such that $x \notin \operatorname{con}(\overline{\mathbf{u}})=\operatorname{con}\left(\overline{\mathbf{u}^{\prime}}\right)$. If $\operatorname{con}(\overline{\mathbf{u}})=\operatorname{con}\left(\overline{\mathbf{u}^{\prime}}\right)=\varnothing$, then the identity $\mathbf{s} \approx \mathbf{s}^{\prime}$ is trivial, so that the result holds with $\Sigma=\varnothing$. Hence, assume that $\operatorname{con}(\overline{\mathbf{u}})=\operatorname{con}\left(\overline{\mathbf{u}^{\prime}}\right) \neq \varnothing$. In what follows, it is shown that identities (3.1) can be used to convert $\mathbf{s}$ into some refined sandwich $\mathbf{r}$. Similarly, identities (3.1) can be used to convert $\mathbf{s}^{\prime}$ into some refined sandwich $\mathbf{r}^{\prime}$. Hence, the equivalence $\left\{(3.1), \mathbf{s} \approx \mathbf{s}^{\prime}\right\} \sim\left\{(3.1), \mathbf{r} \approx \mathbf{r}^{\prime}\right\}$ holds. Since the deduction (3.1) $\vdash \mathbf{s} \approx \mathbf{r}$ holds, it follows from Lemma 3.2 that $\mathbf{s} \approx \mathbf{r} \in \operatorname{id}(\mathbf{L})$, whence $|\operatorname{sim}(\overline{\mathbf{r}})|=|\operatorname{sim}(\overline{\mathbf{s}})|$ and $|\operatorname{non}(\overline{\mathbf{r}})|=|\operatorname{non}(\overline{\mathbf{s}})|$ by Lemma 2.1 .

Let $\mathbf{u}=\prod_{i=1}^{k} \mathbf{p}_{i}$ be the natural decomposition of $\mathbf{u}$, so that $x, \mathbf{p}_{1}, \mathbf{p}_{2}, \ldots, \mathbf{p}_{k}$ are pairwise disjoint and each $\mathbf{p}_{i}$ is either a singleton or a connected word. By Lemma 4.7, identities (3.1) can be used to convert any connected $\mathbf{p}_{i}$ into some sandwich $\mathbf{s}_{i}$ with $\operatorname{sim}\left(\overline{\mathbf{p}_{i}}\right)=\operatorname{sim}\left(\overline{\mathbf{s}_{i}}\right)$ and non $\left(\overline{\mathbf{p}_{i}}\right)=$ non $\left(\overline{\mathbf{s}_{i}}\right)$. Therefore, it can be assumed that $\mathbf{s}$ satisfies (R.1). If $s$ also satisfies (R.2), then $s$ is already refined. Hence, suppose that $s$ does not satisfy (R.2), that is, $\mathbf{p}_{1}, \mathbf{p}_{2}, \ldots, \mathbf{p}_{k}$ are all sandwiches with $k \geq 2$, but $\min \left(\mathbf{p}_{1}\right) \nprec \min \left(\mathbf{p}_{k}\right)$. Then, $\min \left(\mathbf{p}_{k}\right) \prec \min \left(\mathbf{p}_{1}\right)$ because $\mathbf{p}_{1}$ and $\mathbf{p}_{k}$ are disjoint. Let $x_{1}$ be the first letter of $\overline{\mathbf{p}_{i}}$. If $\mathbf{p}_{i}$ is of type (S.1), then $\mathbf{p}_{i}=x_{1} \mathbf{w} x_{1}^{*}$ for some $\mathbf{w} \in\left(\mathscr{A} \cup \mathscr{A}^{*}\right)^{+} \cup\{\varnothing\}$. If $\mathbf{p}_{i}$ is of type (S.2), then $\mathbf{p}_{i}=x_{1}^{*} \mathbf{w} x_{1}$ for some $\mathbf{w} \in\left(\mathscr{A} \cup \mathscr{A}^{*}\right)^{+} \cup\{\varnothing\}$, so that

$$
\mathbf{p}_{i} \stackrel{\text { (inv) }}{\approx} x_{1}^{*} \mathbf{w}\left(x_{1}^{*}\right)^{*} \text {. }
$$

If $\mathbf{p}_{i}$ is of type (S.3), then

$$
\mathbf{p}_{i}=x_{1}^{c_{1}} x_{2}^{c_{2}} \cdots x_{r}^{c_{r}} \mathbf{w} x_{1} x_{2} \cdots x_{r}
$$

for some $\mathbf{w} \in\left(\mathscr{A} \cup \mathscr{A}^{*}\right)^{+} \cup\{\varnothing\}$ and $\mathscr{X}=\left\{x_{1} \prec x_{2} \prec \cdots \prec x_{r}\right\} \subseteq \mathscr{A}$, so that

$$
\mathbf{p}_{i} \stackrel{(3.1 \mathrm{la})}{\approx} x_{1}^{n} \mathbf{p}_{i} \stackrel{(3.1 \mathrm{v})}{\approx} x_{1}^{n} \mathbf{p}_{i} x_{1}^{n} \stackrel{(3.1 \mathrm{~h})}{\approx} x_{1}^{n} \mathbf{p}_{i} x_{1}^{n-\mathbb{R}} x_{1}^{*} \text {. }
$$

Therefore, regardless of type, there exist $h_{i} \in \mathscr{A} \cup \mathscr{A}^{*}$ and $\mathbf{w}_{i} \in\left(\mathscr{A} \cup \mathscr{A}^{*}\right)^{+} \cup\{\varnothing\}$ such that $\mathbf{p}_{i} \stackrel{(3.1)}{\sim} h_{i} \mathbf{w}_{i} h_{i}^{*}$. Hence,

$$
\mathbf{s}=x\left(\prod_{i=1}^{k} \mathbf{p}_{i}\right) x^{*} \stackrel{(3.1)}{\approx} x\left(\prod_{i=1}^{k}\left(h_{i} \mathbf{w}_{i} h_{i}^{*}\right)\right) x^{*} \stackrel{(3.1 \mathrm{w})}{\approx} x\left(\prod_{i=k}^{1}\left(h_{i} \mathbf{w}_{i} h_{i}^{*}\right)\right) x^{*} \stackrel{(3.1)}{\approx} x\left(\prod_{i=k}^{1} \mathbf{p}_{i}\right) x^{*} .
$$

Since $\min \left(\mathbf{p}_{k}\right) \prec \min \left(\mathbf{p}_{1}\right)$, the word $\mathbf{r}=x\left(\prod_{i=k}^{1} \mathbf{p}_{i}\right) x^{*}$ is the required refined sandwich. 
Case 2: The word $s \approx s^{\prime}$ is of type (S.2). Then, by Lemma 5.2,

$$
\mathbf{s}=x^{*} \mathbf{u} x \text { and } \mathbf{s}^{\prime}=x^{*} \mathbf{u}^{\prime} x
$$

for some $x \in \mathscr{A}$ and $\mathbf{u}, \mathbf{u}^{\prime} \in\left(\mathscr{A} \cup \mathscr{A}^{*}\right)^{+} \cup\{\varnothing\}$ such that $x \notin \operatorname{con}(\overline{\mathbf{u}})=\operatorname{con}\left(\overline{\mathbf{u}^{\prime}}\right)$. It is clear that the equivalence $\left\{(3.1), \mathbf{s} \approx \mathbf{s}^{\prime}\right\} \sim\left\{(3.1), x \mathbf{u} x^{*} \approx x \mathbf{u}^{\prime} x^{*}\right\}$ holds. Since $x \mathbf{u} x^{*} \approx x \mathbf{u}^{\prime} x^{*}$ is a sandwich identity of type (S.1), the result follows from Case 1 .

Case 3: The word $s \approx s^{\prime}$ is of type (S.3). Then, by Lemma 5.3,

$$
\mathbf{s}=\mathbf{x} \prod_{i=1}^{\ell}\left(\mathbf{u}_{i} \overrightarrow{\mathscr{X}}\right) \quad \text { and } \quad \mathbf{s}^{\prime}=\mathbf{x} \prod_{i=1}^{\ell}\left(\mathbf{u}_{i}^{\prime} \overrightarrow{\mathscr{X}}\right)
$$

for some $\ell \geq 1$, finite nonempty $\mathscr{X} \subseteq \mathscr{A}, \mathbf{x} \in \mathscr{X}^{\boxplus}$, and $\mathbf{u}_{i}, \mathbf{u}_{i}^{\prime} \in\left(\mathscr{A} \cup \mathscr{A}^{*}\right)^{+} \cup\{\varnothing\}$ such that $\operatorname{con}\left(\overline{\mathbf{u}_{i}}\right)=\operatorname{con}\left(\overline{\mathbf{u}_{i}^{\prime}}\right)$ for each $i$ and $\overrightarrow{\mathscr{X}}, \mathbf{u}_{1}, \mathbf{u}_{2}, \ldots, \mathbf{u}_{\ell}$ are pairwise disjoint. By Lemma 5.4, the equivalence

(A) $\left\{(3.1), \mathbf{s} \approx \mathbf{s}^{\prime}\right\} \sim\{(3.1)\} \cup\left\{x \mathbf{u}_{i} x \approx x \mathbf{u}_{i}^{\prime} x: 1 \leq i \leq \ell\right\}$

holds. It is easily seen that

(B) $\left|\operatorname{sim}\left(\overline{x \mathbf{u}_{i} x}\right)\right| \leq|\operatorname{sim}(\overline{\mathbf{s}})|$ and $\left|\operatorname{non}\left(\overline{x \mathbf{u}_{i} x}\right)\right| \leq|\operatorname{non}(\overline{\mathbf{s}})|$ for all $i$.

For each $i$, the arguments in Case 1 can be repeated to show that the equivalence

(C) $\left\{(3.1), x \mathbf{u}_{i} x \approx x \mathbf{u}_{i}^{\prime} x\right\} \sim\left\{(3.1), \mathbf{r}_{i} \approx \mathbf{r}_{i}^{\prime}\right\}$

holds for some $\mathbf{r}_{i} \approx \mathbf{r}_{i}^{\prime} \in \mathrm{id}_{\mathrm{REF}}\left(\mathbf{L} \times \mathbf{Z}_{n}^{\mathbb{R}}\right)$ such that

(D) $\left|\operatorname{sim}\left(\overline{\mathbf{r}_{i}}\right)\right|=\left|\operatorname{sim}\left(\overline{x \mathbf{u}_{i} x}\right)\right|$ and $\left|\operatorname{non}\left(\overline{\mathbf{r}_{i}}\right)\right|=\left|\operatorname{non}\left(\overline{x \mathbf{u}_{i} x}\right)\right|$.

Hence, the equivalence $\left\{(3.1), \mathbf{s} \approx \mathbf{s}^{\prime}\right\} \sim\{(3.1)\} \cup\left\{\mathbf{r}_{i} \approx \mathbf{r}_{i}^{\prime}: 1 \leq i \leq \ell\right\}$ holds by (A) and (C), where $\left|\operatorname{sim}\left(\overline{\mathbf{r}_{i}}\right)\right| \leq|\operatorname{sim}(\overline{\mathbf{s}})|$ and $\left|\operatorname{non}\left(\overline{\mathbf{r}_{i}}\right)\right| \leq|\operatorname{non}(\overline{\mathbf{s}})|$ by (B) and (D).

Lemma $5.6\left(\left[8\right.\right.$, Lemma 13]). Let $\mathbf{w} \approx \mathbf{w}^{\prime} \in \mathrm{id}(\mathbf{L})$, where $\mathbf{w}, \mathbf{w}^{\prime} \in \mathscr{A}^{+}$. Suppose that $\mathbf{w}$ belongs to

$$
\mathfrak{P}_{k}^{\uparrow}=\left\{x^{q_{1}} y_{1}^{t_{1}} y_{2}^{t_{2}} \cdots y_{k}^{t_{k}} x^{q_{2}}: q_{i} \geq 1, t_{j} \geq 2\right\}
$$

for some $k \geq 2$. Then, $\mathbf{w}^{\prime}$ belongs to either $\mathfrak{P}_{k}^{\uparrow}$ or

$$
\mathfrak{P}_{k}^{\downarrow}=\left\{x^{q_{1}} y_{k}^{t_{k}} y_{k-1}^{t_{k-1}} \cdots y_{1}^{t_{1}} x^{q_{2}}: q_{i} \geq 1, t_{j} \geq 2\right\} .
$$

Lemma 5.7. Let $x \in \mathscr{A}$ and let $\mathbf{s}, \mathbf{s}^{\prime}$ be sandwiches with $x \notin \operatorname{con}(\overline{\mathbf{s}})=\operatorname{con}\left(\overline{\mathbf{s}^{\prime}}\right)$. Suppose that $x \mathbf{s} x \approx x \mathbf{s}^{\prime} x \in \mathrm{id}\left(\mathbf{L} \times \mathbf{Z}_{n}^{\mathbb{R}}\right)$. Then, $\mathbf{s} \approx \mathbf{s}^{\prime} \in \mathrm{id}\left(\mathbf{L} \times \mathbf{Z}_{n}^{\mathbb{R}}\right)$.

Proof. This result has been established for the case $\mathbb{R}=1$ ([10, Lemma 5.8]), but its proof is independent of the value of $\mathbb{R}$. Hence, the present lemma holds.

Lemma 5.8. Suppose that $\mathbf{r} \approx \mathbf{r}^{\prime} \in \mathrm{id}_{\mathrm{REF}}\left(\mathbf{L} \times \mathbf{Z}_{n}^{\mathbb{R}}\right)$, where

$$
\mathbf{r}=x\left(\prod_{i=1}^{k} \mathbf{p}_{i}\right) x^{\circledast}
$$

is the refined sandwich in (5.1). Then,

$$
\mathbf{r}^{\prime}=x\left(\prod_{i=1}^{k} \mathbf{p}_{i}^{\prime}\right) x^{\circledast}
$$

for some $\mathbf{p}_{1}^{\prime}, \mathbf{p}_{2}^{\prime}, \ldots, \mathbf{p}_{k}^{\prime} \in\left(\mathscr{A} \cup \mathscr{A}^{*}\right)^{+}$such that $x, \mathbf{p}_{1}^{\prime}, \mathbf{p}_{2}^{\prime}, \ldots, \mathbf{p}_{k}^{\prime}$ are pairwise disjoint, $\operatorname{con}\left(\overline{\mathbf{p}_{i}}\right)=\operatorname{con}\left(\overline{\mathbf{p}_{i}^{\prime}}\right)$ for all $i$, and each $\mathbf{p}_{i}^{\prime}$ is either a singleton or a sandwich. Furthermore, for each $i$,

(i) $\mathbf{p}_{i}$ and $\mathbf{p}_{i}^{\prime}$ are either both singletons or both sandwiches,

(ii) if $\mathbf{p}_{i}$ and $\mathbf{p}_{i}^{\prime}$ are both singletons, then $\mathbf{p}_{i}=\mathbf{p}_{i}^{\prime}$,

(iii) if $\mathbf{p}_{i}$ and $\mathbf{p}_{i}^{\prime}$ are both sandwiches, then $\mathbf{p}_{i} \approx \mathbf{p}_{i}^{\prime} \in \mathrm{id}_{\mathrm{SAN}}\left(\mathbf{L} \times \mathbf{Z}_{n}^{\mathbb{R}}\right)$.

Proof. It follows from the assumption and Lemmas 5.1-5.3 that $\mathbf{r}^{\prime}=x \mathbf{u}^{\prime} x^{\circledast}$ for some $\mathbf{u}^{\prime} \in\left(\mathscr{A} \cup \mathscr{A}^{*}\right)^{+}$such that (A) $x \notin \operatorname{con}\left(\overline{\mathbf{u}^{\prime}}\right)=\operatorname{con}\left(\overline{\mathbf{p}_{1}} \cdot \overline{\mathbf{p}_{2}} \cdots \overline{\mathbf{p}_{k}}\right)$.

Let $\mathbf{u}^{\prime}=\prod_{i=1}^{k^{\prime}} \mathbf{p}_{i}^{\prime}$ be the natural decomposition of $\mathbf{u}^{\prime}$, so that

$$
\mathbf{r}^{\prime}=x\left(\prod_{i=1}^{k^{\prime}} \mathbf{p}_{i}^{\prime}\right) x^{\circledast},
$$


where

(B) each $\mathbf{p}_{i}^{\prime}$ is either a singleton or a sandwich and $x, \mathbf{p}_{1}^{\prime}, \mathbf{p}_{2}^{\prime}, \ldots, \mathbf{p}_{k^{\prime}}^{\prime}$ are pairwise disjoint.

It is first shown that $k=k^{\prime}$ and $\operatorname{con}\left(\overline{\mathbf{p}_{i}}\right)=\operatorname{con}\left(\overline{\mathbf{p}_{i}^{\prime}}\right)$ for all $i$.

Let $\varphi: \mathscr{A} \rightarrow \mathscr{A}^{+}$denote the substitution given by

$$
z \varphi= \begin{cases}y_{i}^{n} & \text { if } z \in \operatorname{con}\left(\overline{\mathbf{p}_{i}}\right), \\ x^{n} & \text { otherwise. }\end{cases}
$$

Note that for any $\mathbf{w} \in\left(\mathscr{A} \cup \mathscr{A}^{*}\right)^{+}$, the image $\mathbf{w} \varphi$ belongs to

$$
\left(\mathscr{A} \varphi \cup(\mathscr{A} \varphi)^{*}\right)^{+}=\left\{x^{n}, y_{1}^{n}, y_{2}^{n}, \ldots, y_{k}^{n},\left(x^{n}\right)^{*},\left(y_{1}^{n}\right)^{*},\left(y_{2}^{n}\right)^{*}, \ldots,\left(y_{k}^{n}\right)^{*}\right\}^{+} .
$$

Therefore, identity (3.1d) can be used to convert $\mathbf{w} \varphi$ into the plain word $\overline{\mathbf{w} \varphi}$ in $\left\{x^{n}, y_{1}^{n}, y_{2}^{n}, \ldots, y_{k}^{n}\right\}^{+}$. Hence,

$$
\mathbf{r} \varphi \stackrel{(3.1 \mathrm{~d})}{\approx} \overline{\mathbf{r} \varphi}=x^{n}\left(\prod_{i=1}^{k} y_{i}^{n\left|\mathbf{p}_{i}\right|}\right) x^{n} \quad \text { and } \quad \mathbf{r}^{\prime} \varphi \stackrel{(3.1 \mathrm{ld})}{\approx} \overline{\mathbf{r}^{\prime} \varphi}=x^{n}\left(\prod_{i=1}^{k^{\prime}} \overline{\mathbf{p}_{i}^{\prime} \varphi}\right) x^{n} .
$$

Since $\overline{\mathbf{r} \varphi} \approx \overline{\mathbf{r}^{\prime} \varphi} \in \operatorname{id}(\mathbf{L})$ and $\overline{\mathbf{r} \varphi} \in \mathfrak{P}_{k}^{\uparrow}$, Lemma 5.6 implies that $\overline{\mathbf{r}^{\prime} \varphi} \in \mathfrak{P}_{k}^{\uparrow} \cup \mathfrak{P}_{k}^{\downarrow}$, that is, (C) $x^{n}\left(\prod_{i=1}^{k^{\prime}} \overline{\mathbf{p}_{i}^{\prime} \varphi}\right) x^{n} \in \mathfrak{P}_{k}^{\uparrow} \cup \mathfrak{P}_{k}^{\perp}$.

Furthermore, since

$$
\operatorname{con}\left(\overline{\mathbf{p}_{i}^{\prime} \varphi}\right) \subseteq \operatorname{con}\left(\overline{\mathbf{u}^{\prime} \varphi}\right) \stackrel{(\mathrm{A})}{=} \operatorname{con}\left(\overline{\left(\mathbf{p}_{1} \mathbf{p}_{2} \cdots \mathbf{p}_{k}\right) \varphi}\right)=\left\{y_{1}, y_{2}, \ldots, y_{k}\right\}
$$

it follows that

(D) $\operatorname{con}\left(\overline{\mathbf{p}_{i}^{\prime} \varphi}\right) \subseteq\left\{y_{1}, y_{2}, \ldots, y_{k}\right\}$ for each $i \in\left\{1,2, \ldots, k^{\prime}\right\}$.

Recall that each $\mathbf{p}_{i}^{\prime}$ is either a singleton or a sandwich. If $\mathbf{p}_{i}^{\prime}$ is a singleton, then clearly $\operatorname{con}\left(\overline{\mathbf{p}_{i}^{\prime} \varphi}\right)=\left\{y_{j}\right\}$ for some $j$. Suppose that $\mathbf{p}_{i}^{\prime}$ is a sandwich. Then, $\mathbf{p}_{i}^{\prime}$ is connected, so that $\overline{\mathbf{p}_{i}^{\prime} \varphi}$ is a connected factor of $\overline{\mathbf{r}^{\prime} \varphi} \in \mathfrak{P}_{k}^{\uparrow} \cup \mathfrak{P}_{k}^{\downarrow}$. The connected factors of words in $\mathfrak{P}_{k}^{\uparrow} \cup \mathfrak{P}_{k}^{\downarrow}$ are

$$
x^{q_{1}} y_{1}^{t_{1}} y_{2}^{t_{2}} \cdots y_{k}^{t_{k}} x^{q_{2}}, \quad x^{q_{1}} y_{k}^{t_{k}} y_{k-1}^{t_{k-1}} \cdots y_{1}^{t_{1}} x^{q_{2}}, \quad q_{i} \geq 1, t_{j} \geq 2,
$$

and

$$
x^{t}, y_{1}^{t}, y_{2}^{t}, \ldots, y_{k}^{t}, \quad t \geq 2 .
$$

But since $x \notin \operatorname{con}\left(\overline{\mathbf{p}_{i}^{\prime} \varphi}\right)$ by (D), the word $\overline{\mathbf{p}_{i}^{\prime} \varphi}$ can only be one of $y_{1}^{t}, y_{2}^{t}, \ldots, y_{k}^{t}$, so that $\operatorname{con}\left(\overline{\mathbf{p}_{i}^{\prime} \varphi}\right)=\left\{y_{j}\right\}$ for some $j$. Hence, regardless of whether $\mathbf{p}_{i}^{\prime}$ is a singleton or a sandwich,

(E) $\operatorname{con}\left(\overline{\mathbf{p}_{i}^{\prime} \varphi}\right)=\left\{y_{j}\right\}$ for some $j$.

It follows that $\operatorname{con}\left(\overline{\mathbf{p}_{i}^{\prime}}\right) \subseteq \operatorname{con}\left(\overline{\mathbf{p}_{j}}\right)$ for some $j$. By a symmetric argument, the inclusion con $\left(\overline{\mathbf{p}_{j}}\right) \subseteq \operatorname{con}\left(\overline{\mathbf{p}_{m}^{\prime}}\right)$ holds for some $m$, so that $\operatorname{con}\left(\overline{\mathbf{p}_{i}^{\prime}}\right) \subseteq \operatorname{con}\left(\overline{\mathbf{p}_{j}}\right) \subseteq \operatorname{con}\left(\overline{\mathbf{p}_{m}^{\prime}}\right)$. Since the words $\mathbf{p}_{i}^{\prime}$ and $\mathbf{p}_{m}^{\prime}$ are either equal or disjoint, $\mathbf{p}_{i}^{\prime}=\mathbf{p}_{m}^{\prime}$ is the only possibility, whence $\operatorname{con}\left(\overline{\mathbf{p}_{i}^{\prime}}\right)=\operatorname{con}\left(\overline{\mathbf{p}_{j}}\right)$. It has just been shown that for each $i \in\left\{1,2, \ldots, k^{\prime}\right\}$, there exists some $j \in\{1,2, \ldots, k\}$ such that $\operatorname{con}\left(\overline{\mathbf{p}_{i}^{\prime}}\right)=\operatorname{con}\left(\overline{\mathbf{p}_{j}}\right)$. Since $\operatorname{con}\left(\overline{\mathbf{p}_{1}} \cdot \overline{\mathbf{p}_{2}} \cdots \overline{\mathbf{p}_{k}}\right)=\operatorname{con}\left(\overline{\mathbf{p}_{1}^{\prime}} \cdot \overline{\mathbf{p}_{2}^{\prime}} \cdots \overline{\mathbf{p}_{k^{\prime}}^{\prime}}\right)$ by $(\mathrm{A})$, it follows that

(F) $k=k^{\prime}$ and

(G) there exists a one-to-one correspondence between

$$
\operatorname{con}\left(\overline{\mathbf{p}_{1}}\right), \operatorname{con}\left(\overline{\mathbf{p}_{2}}\right), \ldots, \operatorname{con}\left(\overline{\mathbf{p}_{k}}\right) \text { and } \operatorname{con}\left(\overline{\mathbf{p}_{1}^{\prime}}\right), \operatorname{con}\left(\overline{\mathbf{p}_{2}^{\prime}}\right), \ldots, \operatorname{con}\left(\overline{\mathbf{p}_{k}^{\prime}}\right) .
$$

Furthermore, (C) and (F) imply that either

$$
x^{n}\left(\prod_{i=1}^{k} \overline{\mathbf{p}_{i}^{\prime} \varphi}\right) x^{n} \in \mathfrak{P}_{k}^{\uparrow} \quad \text { or } \quad x^{n}\left(\prod_{i=1}^{k} \overline{\mathbf{p}_{i}^{\prime} \varphi}\right) x^{n} \in \mathfrak{P}_{k}^{\downarrow} .
$$

It thus follows from (E) that either

(H) $\left(\operatorname{con}\left(\overline{\mathbf{p}_{1}^{\prime}}\right), \operatorname{con}\left(\overline{\mathbf{p}_{2}^{\prime}}\right), \ldots, \operatorname{con}\left(\overline{\mathbf{p}_{k}^{\prime}}\right)\right)=\left(\operatorname{con}\left(\overline{\mathbf{p}_{1}}\right), \operatorname{con}\left(\overline{\mathbf{p}_{2}}\right), \ldots, \operatorname{con}\left(\overline{\mathbf{p}_{k}}\right)\right)$ or

$\left(\mathrm{H}^{\prime}\right)\left(\operatorname{con}\left(\overline{\mathbf{p}_{1}^{\prime}}\right), \operatorname{con}\left(\overline{\mathbf{p}_{2}^{\prime}}\right), \ldots, \operatorname{con}\left(\overline{\mathbf{p}_{k}^{\prime}}\right)\right)=\left(\operatorname{con}\left(\overline{\mathbf{p}_{k}}\right), \operatorname{con}\left(\overline{\mathbf{p}_{k-1}}\right), \ldots, \operatorname{con}\left(\overline{\mathbf{p}_{1}}\right)\right)$.

Now Lemma 2.1 implies that

(I) $\operatorname{sim}(\overline{\mathbf{r}})=\operatorname{sim}\left(\overline{\mathbf{r}^{\prime}}\right)$ and $\operatorname{non}(\overline{\mathbf{r}})=\operatorname{non}\left(\overline{\mathbf{r}^{\prime}}\right)$.

If $k=1$, then $(\mathrm{H})$ clearly holds, so assume that $k \geq 2$. 
Case 1: The words $\mathbf{p}_{1}, \mathbf{p}_{2}, \ldots, \mathbf{p}_{\boldsymbol{k}}$ are all sandwiches. Then, in view of (B) and (I), either (H) or (H') implies that $\mathbf{p}_{1}^{\prime}, \mathbf{p}_{2}^{\prime}, \ldots, \mathbf{p}_{k}^{\prime}$ are also sandwiches. Hence, (H) must hold by (R.2).

Case 2: The word $\mathbf{p}_{i}$ is a singleton for some $i$. Then, $\mathbf{p}_{i} \in\left\{y, y^{*}\right\}$ for some $y \in \operatorname{sim}(\overline{\mathbf{r}})$. It follows from either $(\mathrm{H})$ or (H') that $\operatorname{con}\left(\overline{\mathbf{p}_{j}^{\prime}}\right)=\{y\}$ for some $j$, whence $\mathbf{p}_{j}^{\prime} \in\left\{y, y^{*}\right\}$ by (I). If $\mathbf{p}_{i} \neq \mathbf{p}_{j}^{\prime}$, so that $\left(\mathbf{p}_{i}, \mathbf{p}_{j}^{\prime}\right) \in\left\{\left(y, y^{*}\right),\left(y^{*}, y\right)\right\}$, then since the group $\mathbf{Z}_{n}^{\mathbb{R}}$ satisfies $\mathbf{r} \approx \mathbf{r}^{\prime}$ and has a unit element, it also satisfies the identity $y^{*} \approx y$; this is impossible because $g^{*}=g^{\mathbb{R}} \neq g$. Therefore, $\mathbf{p}_{i}=\mathbf{p}_{j}^{\prime} \in\left\{y, y^{*}\right\}$. Now since $k \geq 2$, either $1<i$ or $i<k$. By symmetry, assume that $1<i$. Let $\chi: \mathscr{A} \rightarrow L$ denote the substitution given by

$$
z \chi= \begin{cases}e & \text { if } z \in \operatorname{con}\left(\overline{\mathbf{p}_{1}} \cdot \overline{\mathbf{p}_{2}} \cdots \overline{\mathbf{p}_{i-1}}\right) \\ e f & \text { if } z=y \text { and } \mathbf{p}_{i}=y \\ f e & \text { if } z=y \text { and } \mathbf{p}_{i}=y^{*} \\ f & \text { otherwise. }\end{cases}
$$

Then,

$$
\begin{aligned}
\mathbf{r} \chi & =\left(x \cdot \mathbf{p}_{1} \cdot \mathbf{p}_{2} \cdots \mathbf{p}_{i-1} \cdot \mathbf{p}_{i} \cdot \mathbf{p}_{i+1} \cdots \mathbf{p}_{k} \cdot x\right) \chi \\
& =f \cdot e \cdot e \cdots e \cdot e f \cdot f \cdots f \cdot f \\
& =f e f .
\end{aligned}
$$

If ( $\left.\mathrm{H}^{\prime}\right)$ holds, then $\mathbf{r}^{\prime} \chi$ is the product (5.2) in reverse order, that is,

$$
\mathbf{r}^{\prime} \chi=f \cdot f \cdots f \cdot e f \cdot e \cdot e \cdots e \cdot f=0 .
$$

But this is impossible, so that $\left(\mathrm{H}^{\prime}\right)$ cannot hold. Therefore, $(\mathrm{H})$ must hold.

Hence, (H) holds in any case. It then follows from (B) and (I) that (i) and (ii) hold. It remains to verify that (iii) also holds. Suppose that $\mathbf{p}_{i}$ and $\mathbf{p}_{i}^{\prime}$ are sandwiches. Let $\psi: \mathscr{A} \rightarrow \mathscr{A}$ denote the substitution given by

$$
z \psi= \begin{cases}z & \text { if } z \in \operatorname{con}\left(\overline{\mathbf{p}_{i}}\right)=\operatorname{con}\left(\overline{\mathbf{p}_{i}^{\prime}}\right), \\ x^{n} & \text { otherwise. }\end{cases}
$$

Then, the deductions (3.1a) $\vdash\left\{x(\mathbf{r} \psi) x \approx x \mathbf{p}_{i} x, x\left(\mathbf{r}^{\prime} \psi\right) x \approx x \mathbf{p}_{i}^{\prime} x\right\}$ hold, so that the deduction $\left\{(3.1 \mathrm{a}), \mathbf{r} \approx \mathbf{r}^{\prime}\right\} \vdash$ $x \mathbf{p}_{i} x \approx x \mathbf{p}_{i}^{\prime} x$ also holds. It now follows from Lemma 3.2 that $x \mathbf{p}_{i} x \approx x \mathbf{p}_{i}^{\prime} x \in \mathrm{id}\left(\mathbf{L} \times \mathbf{Z}_{n}^{\mathbb{R}}\right)$. Hence, $\mathbf{p}_{i} \approx \mathbf{p}_{i}^{\prime} \in \mathrm{id}\left(\mathbf{L} \times \mathbf{Z}_{n}^{\mathbb{R}}\right)$ by Lemma 5.7 .

\section{Proof of Theorem 3.1}

In this section, the following statement is established for each $m \geq 1$.

$\left(\S_{m}\right)$ If $\mathbf{s} \approx \mathbf{s}^{\prime} \in \mathrm{id}_{\mathrm{SAN}}\left(\mathbf{L} \times \mathbf{Z}_{n}^{\mathbb{R}}\right)$ with $|\operatorname{non}(\overline{\mathbf{s}})| \leq m$, then $(3.1) \vdash \mathbf{s} \approx \mathbf{s}^{\prime}$.

Hence, the deduction (3.1) $\vdash \mathrm{id}_{\mathrm{SAN}}\left(\mathbf{L} \times \mathbf{Z}_{n}^{\mathbb{R}}\right)$ holds. It follows from Lemma 4.4 that the deduction

$$
\{(3.1)\} \cup \operatorname{id}_{\mathrm{SAN}}\left(\mathbf{L} \times \mathbf{Z}_{n}^{\mathbb{R}}\right) \vdash \mathrm{id}\left(\mathbf{L} \times \mathbf{Z}_{n}^{\mathbb{R}}\right)
$$

holds. Therefore, the proof of Theorem 3.1 is complete.

Lemma 6.1. The statement $\left(\S_{1}\right)$ holds.

Proof. Suppose that $\mathbf{s} \approx \mathbf{s}^{\prime} \in \mathrm{id}_{\mathrm{SAN}}\left(\mathbf{L} \times \mathbf{Z}_{n}^{\mathbb{R}}\right)$ with $|\operatorname{non}(\overline{\mathbf{s}})|=1$. Then, by Lemma 5.5, there exists some finite $\Sigma \subseteq \operatorname{id}_{\operatorname{REF}}\left(\mathbf{L} \times \mathbf{Z}_{n}^{\mathbb{R}}\right)$ such that

(A) $\left\{(3.1), \mathbf{s} \approx \mathbf{s}^{\prime}\right\} \sim\{(3.1)\} \cup \Sigma$ and

(B) each $\mathbf{r} \approx \mathbf{r}^{\prime} \in \Sigma$ satisfies $|\operatorname{sim}(\overline{\mathbf{r}})| \leq|\operatorname{sim}(\overline{\mathbf{s}})|$ and $|\operatorname{non}(\overline{\mathbf{r}})| \leq|\operatorname{non}(\overline{\mathbf{s}})|=1$.

Consider any $\mathbf{r} \approx \mathbf{r}^{\prime} \in \Sigma$. Generality is not lost by assuming that $\mathbf{r}=x\left(\prod_{i=1}^{k} \mathbf{p}_{i}\right) x^{\circledast}$ is the refined sandwich in (5.1). Then, Lemma 5.8 implies that $\mathbf{r}^{\prime}=x\left(\prod_{i=1}^{k} \mathbf{p}_{i}^{\prime}\right) x^{\circledast}$ for some $\mathbf{p}_{i}^{\prime} \in\left(\mathscr{A} \cup \mathscr{A}^{*}\right)^{+}$such that $\operatorname{con}\left(\overline{\mathbf{p}_{i}}\right)=\operatorname{con}\left(\overline{\mathbf{p}_{i}^{\prime}}\right)$ 
for all $i$. Since $1=|\operatorname{non}(\overline{\mathbf{r}})|=\left|\operatorname{non}\left(\overline{\mathbf{r}^{\prime}}\right)\right|$ by (B) and Lemma 2.1, it follows from (i) and (ii) of Lemma 5.8 that $\mathbf{p}_{i}$ and $\mathbf{p}_{i}^{\prime}$ are the same singleton. Hence, the identity $\mathbf{r} \approx \mathbf{r}^{\prime}$ is trivial.

Since the identity $\mathbf{r} \approx \mathbf{r}^{\prime}$ is arbitrary in $\Sigma$, every identity in $\Sigma$ is trivial. The deduction (3.1) $\vdash \mathbf{s} \approx \mathbf{s}^{\prime}$ thus follows from (A).

Lemma 6.2. Suppose that the statement $\left(\S_{m}\right)$ holds. Then, the statement $\left(\S_{m+1}\right)$ also holds.

Proof. Suppose that $\mathbf{s} \approx \mathbf{s}^{\prime} \in \mathrm{id}_{\mathrm{SAN}}\left(\mathbf{L} \times \mathbf{Z}_{n}^{\mathbb{R}}\right)$ with $|\operatorname{non}(\mathbf{s})|=m+1$. Then, by Lemma 5.5, there exists some finite $\Sigma \subseteq \operatorname{id}_{\mathrm{REF}}\left(\mathbf{L} \times \mathbf{Z}_{n}^{\mathbb{R}}\right)$ such that

(A) $\left\{(3.1), \mathbf{s} \approx \mathbf{s}^{\prime}\right\} \sim\{(3.1)\} \cup \Sigma$ and

(B) each $\mathbf{r} \approx \mathbf{r}^{\prime} \in \Sigma$ satisfies $|\operatorname{sim}(\overline{\mathbf{r}})| \leq|\operatorname{sim}(\overline{\mathbf{s}})|$ and $|\operatorname{non}(\overline{\mathbf{r}})| \leq|\operatorname{non}(\overline{\mathbf{s}})|=m+1$.

Consider any $\mathbf{r} \approx \mathbf{r}^{\prime} \in \Sigma$. Generality is not lost by assuming that $\mathbf{r}=x\left(\prod_{i=1}^{k} \mathbf{p}_{i}\right) x^{\circledast}$ is the refined sandwich in (5.1). Then, Lemma 5.8 implies that $\mathbf{r}^{\prime}=x\left(\prod_{i=1}^{k} \mathbf{p}_{i}^{\prime}\right) x^{\circledast}$ for some $\mathbf{p}_{i}^{\prime} \in\left(\mathscr{A} \cup \mathscr{A}^{*}\right)^{+}$such that $\operatorname{con}\left(\overline{\mathbf{p}_{i}}\right)=\operatorname{con}\left(\overline{\mathbf{p}_{i}^{\prime}}\right)$ for all $i$. By (i) of Lemma 5.8, the words $\mathbf{p}_{i}$ and $\mathbf{p}_{i}^{\prime}$ are both singletons or both sandwiches.

Case 1: The words $\mathbf{p}_{i}$ and $\mathbf{p}_{i}^{\prime}$ are singletons. Then, one has $\mathbf{p}_{i}=\mathbf{p}_{i}^{\prime}$ by (ii) of Lemma 5.8, so that the deduction (3.1) $\vdash \mathbf{p}_{i} \approx \mathbf{p}_{i}^{\prime}$ holds vacuously.

Case 2: The words $\mathbf{p}_{i}$ and $\mathbf{p}_{i}^{\prime}$ are both sandwiches. Then, $\mathbf{p}_{i} \approx \mathbf{p}_{i}^{\prime} \in \mathrm{id}_{\mathrm{SAN}}\left(\mathbf{L} \times \mathbf{Z}_{n}^{\mathbb{R}}\right)$ by (iii) of Lemma 5.8. Since $\left|\operatorname{non}\left(\overline{\mathbf{p}_{i}}\right)\right|<|\operatorname{non}(\overline{\mathbf{r}})| \leq m+1$ by (B), the deduction (3.1) $\vdash \mathbf{p}_{i} \approx \mathbf{p}_{i}^{\prime}$ follows from $\left(\S_{m}\right)$.

Therefore, the deduction (3.1) $\vdash \mathbf{p}_{i} \approx \mathbf{p}_{i}^{\prime}$ holds in any case. Since

$$
\mathbf{r}=x\left(\prod_{i=1}^{k} \mathbf{p}_{i}\right) x^{\circledast} \stackrel{(3.1)}{\sim} x\left(\prod_{i=1}^{k} \mathbf{p}_{i}^{\prime}\right) x^{\circledast}=\mathbf{r}^{\prime},
$$

the deduction (3.1) $\vdash \mathbf{r} \approx \mathbf{r}^{\prime}$ also holds. The identity $\mathbf{r} \approx \mathbf{r}^{\prime}$ is arbitrary in $\Sigma$, so that (3.1) $\vdash \Sigma$. Consequently, the deduction (3.1) $\vdash \mathbf{s} \approx \mathbf{s}^{\prime}$ follows from (A).

\section{Irredundant basis property}

\subsection{Terms, identities, and deducibility}

The set $T_{\mathscr{A}}$ of terms over $\mathscr{A}$ is the smallest set that satisfies all of the following:

- $\mathscr{A} \subseteq T_{\mathscr{A}}$,

- if $\mathbf{t}_{1}, \mathbf{t}_{2} \in T_{\mathscr{A}}$, then $\mathbf{t}_{1} \mathbf{t}_{2} \in T_{\mathscr{A}}$,

- if $\mathbf{t} \in T_{\mathscr{A}}$, then $\mathbf{t}^{*} \in T_{\mathscr{A}}$.

The subterms of a term $\mathbf{t}$ are defined as follows:

- $t$ is a subterm of $t$,

- if $\mathbf{t}_{1} \mathbf{t}_{2}$ is a subterm of $\mathbf{t}$, where $\mathbf{t}_{1}, \mathbf{t}_{2} \in T_{\mathscr{A}}$, then $\mathbf{t}_{1}$ and $\mathbf{t}_{2}$ are subterms of $\mathbf{t}$,

- if $\mathbf{u}^{*}$ is a subterm of $\mathbf{t}$, where $\mathbf{u} \in T_{\mathscr{A}}$, then $\mathbf{u}$ is a subterm of $\mathbf{t}$.

Remark 7.1. Note the following.

(i) The inclusion $\left(\mathscr{A} \cup \mathscr{A}^{*}\right)^{+} \subset T_{\mathscr{A}}$ holds.

(ii) The identities (inv) can be used to convert any term $\mathbf{t} \in T_{\mathscr{A}}$ into a unique word in $\left(\mathscr{A} \cup \mathscr{A}^{*}\right)^{+}$; denote this unique word by $\lfloor\mathbf{t}\rfloor$.

(iii) If $\mathbf{u}$ is a subterm of a term $\mathbf{t} \in T_{\mathscr{A}}$, then either $\lfloor\mathbf{u}\rfloor$ or $\left\lfloor\mathbf{u}^{*}\right\rfloor$ is a factor of the word $\lfloor\mathbf{t}\rfloor$.

For the remainder of this article, identities $\mathbf{t} \approx \mathbf{t}^{\prime}$ are formed by terms $\mathbf{t}, \mathbf{t}^{\prime} \in T_{\mathscr{A}}$. An identity $\mathbf{w} \approx \mathbf{w}^{\prime}$ formed by words $\mathbf{w}, \mathbf{w}^{\prime} \in\left(\mathscr{A} \cup \mathscr{A}^{*}\right)^{+}$is called a word identity. The objective of Sections 3-6 was to prove that identities (3.1) constitute a basis for the involution semigroup $\mathbf{L} \times \mathbf{Z}_{n}^{\mathbb{R}}$. In view of (ii) of Remark 7.1, this task was achievable by working mainly with word identities. However, the situation is different in the present section, where the goal is to

(‡) extract from (3.1) an infinite irredundant basis for $\mathbf{L} \times \mathbf{Z}_{n}^{\mathbb{R}}$; 
see Theorem 7.4. Each identity from this extracted basis is shown to be undeducible from all other identities in the basis, and this involves examining deduction sequences that generally contain terms instead of only words. Working with only word identities is thus insufficient.

Due to ( $¥$ ), the concept of deducibility of identities, first defined in Section 2, has to be treated more formally. An identity $\mathbf{x} \approx \mathbf{y}$ is directly deducible from an identity $\mathbf{u} \approx \mathbf{v}$ if there exists some substitution $\varphi: \mathscr{A} \rightarrow T_{\mathscr{A}}$ such that $\mathbf{u} \varphi$ is a subterm of $\mathbf{x}$, and replacing this subterm of $\mathbf{x}$ with $\mathbf{v} \varphi$ results in the term $\mathbf{y}$. By Birkhoff's completeness theorem of equational logic [2], an identity $\mathbf{x} \approx \mathbf{y}$ is deducible from a set $\Sigma$ of identities if there exists a sequence

$$
\mathbf{x}=\mathbf{t}_{1}, \mathbf{t}_{2}, \ldots, \mathbf{t}_{r}=\mathbf{y}
$$

of terms, where each identity $\mathbf{t}_{i} \approx \mathbf{t}_{i+1}$ is directly deducible from some identity in $\Sigma$.

Recall that the number of times a letter $x \in \mathscr{A}$ occurs in a word $\mathbf{w} \in\left(\mathscr{A} \cup \mathscr{A}^{*}\right)^{+}$is denoted by occ $(x, \overline{\mathbf{w}})$. The number of times $x \in \mathscr{A}$ occurs in a term $\mathbf{t} \in T_{\mathscr{A}}$ is $\operatorname{occ}(x, \overline{[\mathbf{t}]})$. For instance, if $\mathbf{t}=x\left(y^{*} x^{2}\right)^{*}\left(z x^{*} y\right)^{*} \in T_{\mathscr{A}}$, then

$$
\overline{\lfloor\mathbf{t}\rfloor}=\overline{x\left(x^{*}\right)^{2} y y^{*} x z^{*}}=x^{3} y^{2} x z,
$$

so that $\operatorname{occ}(x, \overline{\lfloor\mathbf{t}\rfloor})=4, \operatorname{occ}(y, \overline{\mathbf{t}\rfloor})=2$, and $\operatorname{occ}(z, \overline{\mathbf{t}\rfloor})=1$. The following result is easily seen to hold.

Lemma 7.2. Suppose that $\mathbf{t} \approx \mathbf{t}^{\prime}$ is any identity deducible from (inv). Then, one has $\lfloor\mathbf{t}\rfloor=\left\lfloor\mathbf{t}^{\prime}\right\rfloor$.

\subsection{Identities (3.1w)}

For each $m \geq 2$, let

$$
\mathbb{B}_{m}=\left\{\left(b_{1}, b_{2}, \ldots, b_{m}\right): b_{1}, b_{2}, \ldots, b_{m} \in\{0,1\}\right\}
$$

denote the set of all binary vectors of dimension $m$. The vectors in $\mathbb{B}_{m}$ are lexicographically ordered by $<$ as

$$
\left(b_{1}, b_{2}, \ldots, b_{m}\right)<\left(b_{1}^{\prime}, b_{2}^{\prime}, \ldots, b_{m}^{\prime}\right)
$$

if there exists a least $\ell \in\{1,2, \ldots, m\}$ such that $b_{\ell}<b_{\ell}^{\prime}$ and $b_{i}=b_{i}^{\prime}$ for any $i<\ell$. If $\mathrm{v}=\left(b_{1}, b_{2}, \ldots, b_{m}\right) \in \mathbb{B}_{m}$, then the dual of $\mathrm{V}$ is $\delta \mathrm{V}=\left(b_{m}, b_{m-1}, \ldots, b_{1}\right)$. If $\delta \mathrm{V}=\mathrm{V}$, then $\mathrm{V}$ is a palindrome. The set $\mathbb{B}_{m}$ can be partitioned into the three subsets

$$
\mathbb{B}_{m}^{=}=\left\{\mathrm{v} \in \mathbb{B}_{m}: \mathrm{V}=\delta \mathrm{v}\right\}, \quad \mathbb{B}_{m}^{<}=\left\{\mathrm{V} \in \mathbb{B}_{m}: \mathrm{v}<\delta \mathrm{v}\right\}, \quad \mathbb{B}_{m}^{>}=\left\{\mathrm{v} \in \mathbb{B}_{m}: \mathrm{V}>\delta \mathrm{v}\right\} .
$$

Each vector $\mathrm{V}=\left(b_{1}, b_{2}, \ldots, b_{m}\right) \in \mathbb{B}_{m}$ is associated with the words

$$
\begin{aligned}
& \mathrm{V}^{\uparrow}=x\left(\prod_{i=1}^{m}\left(y_{i} h_{i}^{b_{i}} y_{i}^{*}\right)\right) x^{*}=x \cdot y_{1} h_{1}^{b_{1}} y_{1}^{*} \cdot y_{2} h_{2}^{b_{2}} y_{2}^{*} \cdots y_{m} h_{m}^{b_{m}} y_{m}^{*} \cdot x^{*}, \\
& \mathrm{~V}^{\downarrow}=x\left(\prod_{i=m}^{1}\left(y_{i} h_{i}^{b_{i}} y_{i}^{*}\right)\right) x^{*}=x \cdot y_{m} h_{m}^{b_{m}} y_{m}^{*} \cdot y_{m-1} h_{m-1}^{b_{m-1}} y_{m-1}^{*} \cdots y_{1} h_{1}^{b_{1}} y_{1}^{*} \cdot x^{*},
\end{aligned}
$$

where $h_{i}^{0}=\varnothing$ and $h_{i}^{1}=h_{i}$. Note that

$$
\bigcup_{m \geq 2}\left\{\mathrm{~V}^{\uparrow} \approx \mathrm{V}^{\downarrow}: \mathrm{V} \in \mathbb{B}_{m}\right\}=\{(3.1 \mathrm{w})\} .
$$

In Section 7.3, some identities from (3.1w) are chosen to form an irredundant basis for the involution semigroup $\mathbf{L} \times \mathbf{Z}_{n}^{\mathbb{R}}$. But for each $m \geq 2$, the identities in $\left\{\mathrm{V}^{\uparrow} \approx \mathrm{V}^{\downarrow}: \mathrm{V} \in \mathbb{B}_{m}\right\}$ are not irredundant; if $\mathrm{V}$ is not a palindrome, then $\mathrm{V}$ and $\delta \mathrm{V}$ are distinct vectors such that the associated identities $\mathrm{V}^{\uparrow} \approx \mathrm{V}^{\downarrow}$ and $(\delta \mathrm{V})^{\uparrow} \approx(\delta \mathrm{V})^{\downarrow}$ are equivalent. This redundancy can be eliminated by choosing identities $\mathrm{V}^{\uparrow} \approx \mathrm{V}^{\downarrow}$ with $\mathrm{V}$ taken from only $\mathbb{B}_{m}^{=}$ or $\mathbb{B}_{m}^{<}$. Hence, the equivalence

$$
\text { (3.1w) } \sim \bigcup_{m \geq 2}\left\{\mathrm{~V}^{\uparrow} \approx \mathrm{V}^{\downarrow}: \mathrm{V} \in \mathbb{B}_{m}^{=} \cup \mathbb{B}_{m}^{<}\right\}
$$

holds, where no two identities on the right are equivalent. 
Lemma 7.3. Let $\mathrm{v}=\left(b_{1}, b_{2}, \ldots, b_{m}\right) \in \mathbb{B}_{m}$. Suppose that $\mathrm{v}^{\uparrow} \approx \mathbf{w} \in \mathrm{id}\left(\mathbf{L} \times \mathbf{Z}_{n}^{\mathbb{R}}\right)$ for some word $\mathbf{w} \in\left(\mathscr{A} \cup \mathscr{A}^{*}\right)^{+}$. Then, either $\mathbf{w}=\mathrm{v}^{\uparrow}$ or $\mathbf{w}=\mathrm{V}^{\downarrow}$.

Proof. By Lemma 2.1,

(A) $\operatorname{sim}(\overline{\mathbf{w}})=\left\{h_{1}^{b_{1}}, h_{2}^{b_{2}}, \ldots, h_{m}^{b_{m}}\right\}$ and $\operatorname{non}(\overline{\mathbf{w}})=\left\{x, y_{1}, y_{2}, \ldots, y_{m}\right\}$.

Let $\varphi: \mathscr{A} \rightarrow L$ denote the substitution given by

$$
z \varphi= \begin{cases}f e & \text { if } z=x, \\ e & \text { otherwise }\end{cases}
$$

Then, $\mathrm{v}^{\uparrow} \varphi=f e f=\mathbf{w} \varphi$, so that $\mathbf{w} \varphi$ is a product of the form $f e \cdot e \cdot e \cdot \cdots e \cdot e f$, whence

(B) $\mathbf{w}=x \mathbf{u} x^{*}$ for some $\mathbf{u} \in\left(\mathscr{A} \cup \mathscr{A}^{*}\right)^{+}$such that $x \notin \operatorname{con}(\overline{\mathbf{u}})$.

Consider any fixed $i \in\{1,2, \ldots, m\}$. Let $\chi: \mathscr{A} \rightarrow L$ denote the substitution given by

$$
z \chi= \begin{cases}f e & \text { if } z=y_{i}, \\ e & \text { if } z=h_{i}, \\ f & \text { otherwise. }\end{cases}
$$

Then, $\mathrm{v}^{\uparrow} \chi=f e f=\mathbf{w} \chi$.

There are two cases to consider.

Case 1. If $b_{i}=1$, then $h_{i} \in \operatorname{sim}(\overline{\mathrm{w}})$ by (A), so that

$$
\mathbf{w} \chi=f \cdot f \cdots f \cdot f e \cdot e \cdot e f \cdot f \cdot f \cdots f .
$$

Hence, (A) and (B) imply that $\mathbf{w}=x \mathbf{u}_{1} y_{i} h_{i}^{\circledast} y_{i}^{*} \mathbf{u}_{2} x^{*}$ for some $\circledast \in\{1, *\}$ and $\mathbf{u}_{1}, \mathbf{u}_{2} \in\left(\mathscr{A} \cup \mathscr{A}^{*}\right)^{+} \cup\{\varnothing\}$ such that $x, y_{i}, h_{i} \notin \operatorname{con}\left(\overline{\mathbf{u}_{1}} \cdot \overline{\mathbf{u}_{2}}\right)$. Since $\mathrm{v}^{\uparrow} \approx \mathbf{w} \in \operatorname{id}\left(\mathbf{Z}_{n}^{\mathbb{R}}\right)$ and $\mathbf{Z}_{n}^{\mathbb{R}}$ has a unit element, it follows that $h_{i} \approx h_{i}^{\circledast} \in \operatorname{id}\left(\mathbf{Z}_{n}^{\mathbb{R}}\right)$. But $g \neq g^{*}$ in $\mathbf{Z}_{n}^{\mathbb{R}}$, so that $\circledast=1$. Thus $\mathbf{w}=x \mathbf{u}_{1} y_{i} h_{i}^{1} y_{i}^{*} \mathbf{u}_{2} x^{*}=x \mathbf{u}_{1} y_{i} h_{i}^{b_{i}} y_{i}^{*} \mathbf{u}_{2} x^{*}$.

Case 2. If $b_{i}=0$, then $h_{i} \notin \operatorname{con}(\overline{\mathbf{w}})$ by (A), so that

$$
\mathbf{w} \chi=f \cdot f \cdots f \cdot f e \cdot e f \cdot f \cdot f \cdots f .
$$

Hence, (A) and (B) imply that $\mathbf{w}=x \mathbf{u}_{1} y_{i} y_{i}^{*} \mathbf{u}_{2} x^{*}=x \mathbf{u}_{1} y_{i} h_{i}^{b_{i}} y_{i}^{*} \mathbf{u}_{2} x^{*}$ for some $\mathbf{u}_{1}, \mathbf{u}_{2} \in\left(\mathscr{A} \cup \mathscr{A}^{*}\right)^{+} \cup\{\varnothing\}$ such that $x, y_{i} \notin \operatorname{con}\left(\overline{\mathbf{u}_{1}} \cdot \overline{\mathbf{u}_{2}}\right)$.

Therefore, in any case, $\mathbf{w}=x \mathbf{u}_{1} y_{i} h_{i}^{b_{i}} y_{i}^{*} \mathbf{u}_{2} x^{*}$ for some $\mathbf{u}_{1}, \mathbf{u}_{2} \in\left(\mathscr{A} \cup \mathscr{A}^{*}\right)^{+} \cup\{\varnothing\}$ such that $x, y_{i}, h_{i} \notin \operatorname{con}\left(\overline{\mathbf{u}_{1}} \cdot \overline{\mathbf{u}_{2}}\right)$. Since $i$ is arbitrary, it follows that $\mathbf{w}=x \mathbf{v} x^{*}$, where $\mathbf{v}$ is a product of $y_{1} h_{1}^{b_{1}} y_{1}^{*}, y_{2} h_{2}^{b_{2}} y_{2}^{*}, \ldots, y_{m} h_{m}^{b_{m}} y_{m}^{*}$ in some order. Let $\pi$ denote the permutation on $\{1,2, \ldots, m\}$ such that

$$
\mathbf{w}=x\left(\prod_{i=1}^{m}\left(y_{i \pi} h_{i \pi}^{b_{i \pi}} y_{i \pi}^{*}\right)\right) x^{*} .
$$

Let $\psi: \mathscr{A} \rightarrow \mathscr{A}^{+}$denote the substitution given by

$$
z \psi= \begin{cases}y_{i}^{n} & \text { if } z \in\left\{y_{i}, h_{i}\right\}, \\ x^{n} & \text { otherwise. }\end{cases}
$$

Then,

$$
\mathrm{V}^{\uparrow} \psi \stackrel{(3.1 \mathrm{a}),(3.1 \mathrm{~d})}{\approx} x^{n}\left(\prod_{i=1}^{m} y_{i}^{n}\right) x^{n} \in \mathfrak{P}_{m}^{\uparrow} \text { and } \mathbf{w} \psi \stackrel{(3.1 \mathrm{a}),(3.1 \mathrm{~d})}{\approx} x^{n}\left(\prod_{i=1}^{m} y_{i \pi}^{n}\right) x^{n} .
$$

Since $x^{n}\left(\prod_{i=1}^{m} y_{i}^{n}\right) x^{n} \approx x^{n}\left(\prod_{i=1}^{m} y_{i \pi}^{n}\right) x^{n} \in \mathrm{id}\left(\mathbf{L} \times \mathbf{Z}_{n}^{\mathbb{R}}\right)$, it follows from Lemma 5.6 that $x^{n}\left(\prod_{i=1}^{m} y_{i \pi}^{n}\right) x^{n} \in \mathfrak{P}_{m}^{\uparrow} \cup \mathfrak{P}_{m}^{\downarrow}$, whence

$$
(1 \pi, 2 \pi, \ldots, m \pi) \in\{(1,2, \ldots, m),(m, m-1, \ldots, 1)\} .
$$

Consequently, either $\mathbf{w}=\mathrm{v}^{\uparrow}$ or $\mathbf{w}=\mathrm{V}^{\downarrow}$. 


\subsection{An irredundant basis from (3.1)}

Theorem 7.4. An infinite irredundant basis for the involution semigroup $\mathbf{L} \times \mathbf{Z}_{n}^{\mathbb{R}}$ can be formed from the identities in (3.1).

Recall from Section 7.2 that identities (3.1w) and

$$
\Omega=\bigcup_{m \geq 2}\left\{\mathrm{~V}^{\uparrow} \approx \mathrm{V}^{\downarrow}: \mathrm{V} \in \mathbb{B}_{m}^{=} \cup \mathbb{B}_{m}^{<}\right\}
$$

are equivalent. Since the set $\Theta=\{(3.1 \mathrm{a}),(3.1 \mathrm{~b}), \ldots,(3.1 \mathrm{v})\}$ is finite, it follows from Theorem 3.1 that $\Theta$ contains some minimal subset $\Theta_{\min }$ such that $\Theta_{\min } \cup \Omega$ is a basis for the involution semigroup $\mathbf{L} \times \mathbf{Z}_{n}^{\mathbb{R}}$. Let

$$
\mathrm{V}_{\text {fix }}=\left(b_{1}, b_{2}, \ldots, b_{m}\right) \in \mathbb{B}_{m}^{=} \cup \mathbb{B}_{m}^{<}
$$

be a fixed vector. In the remainder of this subsection, it is shown that the identity

$$
\mathrm{V}_{\text {fix }}^{\uparrow} \approx \mathrm{V}_{\text {fix }}^{\downarrow}
$$

in $\Omega$ is not deducible from

$$
\{(\text { inv })\} \cup \Theta_{\text {min }} \cup\left(\Omega \backslash\left\{\mathrm{v}_{\text {fix }}^{\uparrow} \approx \mathrm{v}_{\text {fix }}^{\downarrow}\right\}\right) .
$$

Since $\Omega$ is infinite, the set $\Theta_{\min } \cup \Omega$ is an infinite irredundant basis for the involution semigroup $\mathbf{L} \times \mathbf{Z}_{n}^{\mathbb{R}}$. This completes the proof of Theorem 7.4.

Seeking a contradiction, suppose that the identity $v_{\text {fix }}^{\uparrow} \approx v_{\text {fix }}^{\downarrow}$ is deducible from identities (7.1). Then, there exists a sequence

$$
\mathrm{V}_{\text {fix }}^{\uparrow}=\mathbf{t}_{1}, \mathbf{t}_{2}, \ldots, \mathbf{t}_{r}=\mathrm{V}_{\text {fix }}^{\downarrow}
$$

of terms, where each identity $\mathbf{t}_{i} \approx \mathbf{t}_{i+1}$ is directly deducible from some identity $\mathbf{u}_{i} \approx \mathbf{v}_{i}$ in (7.1). If every identity $\mathbf{u}_{i} \approx \mathbf{v}_{i}$ is from (inv), then it follows from Lemma 7.2 that $\mathrm{v}_{\text {fix }}^{\uparrow}=\left\lfloor\mathbf{t}_{i}\right\rfloor$ for all $i$, whence the contradiction $\mathrm{V}_{\text {fix }}^{\uparrow}=\left\lfloor\mathbf{t}_{r}\right\rfloor=\mathrm{V}_{\text {fix }}^{\downarrow}$ is obtained. Therefore, some $\mathbf{u}_{i} \approx \mathbf{v}_{i}$ is not from (inv); let $\ell \geq 1$ be the least possible index such that $\mathbf{u}_{\ell} \approx \mathbf{v}_{\ell}$ is not from (inv). Then,

(I) $\mathbf{u}_{\ell} \approx \mathbf{v}_{\ell}$ is from $\Theta_{\text {min }} \cup\left(\Omega \backslash\left\{\mathrm{v}_{\text {fix }}^{\uparrow} \approx \mathrm{v}_{\text {fix }}^{\downarrow}\right\}\right)$

while $\mathbf{u}_{1} \approx \mathbf{v}_{1}, \mathbf{u}_{2} \approx \mathbf{v}_{2}, \ldots, \mathbf{u}_{\ell-1} \approx \mathbf{v}_{\ell-1}$ are from (inv), whence

(II) $\mathrm{V}_{\text {fix }}^{\uparrow} \approx \mathbf{t}_{\ell}$ is deducible from (inv).

In what follows, it is shown that the identity $\mathbf{u}_{\ell} \approx \mathbf{v}_{\ell}$ belongs to neither $\Theta_{\min }$ nor $\Omega \backslash\left\{\mathrm{v}_{\text {fix }}^{\uparrow} \approx \mathrm{V}_{\text {fix }}^{\downarrow}\right\}$; this and (I) imply the required contradiction.

Lemma 7.5. The following hold.

(i) $\left\lfloor\mathbf{t}_{\ell}\right\rfloor=\mathrm{V}_{\text {fix }}^{\uparrow}$.

(ii) The word $\left\lfloor\mathbf{t}_{\ell}\right\rfloor$ does not contain any of the factors

$$
\begin{aligned}
& x^{\circledast_{1}} \mathbf{h}_{1} x^{\circledast_{1}}, \\
& x^{\circledast_{1}} \mathbf{h}_{1} x^{\circledast_{2}} \mathbf{h}_{2} x^{\circledast_{3}}, \\
& x^{\circledast_{1}} \mathbf{h}_{1} y^{\circledast_{2}} \mathbf{h}_{2} x^{\circledast_{3}} \mathbf{h}_{3} y^{\oplus_{4}}, \\
& x^{\circledast_{1}} \mathbf{h}_{1} y^{\otimes_{2}} \mathbf{h}_{2} z^{\circledast_{3}} \mathbf{h}_{3} z^{\otimes_{4}} \mathbf{h}_{4} y^{\otimes_{5}} \mathbf{h}_{5} x^{\circledast_{6}} \text {, } \\
& x^{\circledast_{1}} \mathbf{h}_{1} y^{\circledast_{2}} \mathbf{h}_{2} z^{\circledast_{3}} t^{\circledast_{4}} \mathbf{h}_{3} y^{\circledast_{5}} \mathbf{h}_{4} x^{\circledast_{6}},
\end{aligned}
$$

where $x, y, z, t \in \mathscr{A}, \mathbf{h}_{i} \in\left(\mathscr{A} \cup \mathscr{A}^{*}\right)^{+} \cup\{\varnothing\}$, and $\circledast_{i} \in\{1, *\}$.

[Correction added after online publication 11 September 2015: For the readers convenience equation (7.2a) has been changed from $x^{\circledast_{1}} \mathbf{h}_{1} x^{\circledast_{2}}$ to $x^{\circledast_{1}} \mathbf{h}_{1} x^{\circledast_{1}}$.]

Proof. Part (i) follows from (II) and the observation made in (ii) of Remark 7.1, while part (ii) is a consequence of part (i).

Since the identity $\mathbf{t}_{\ell} \approx \mathbf{t}_{\ell+1}$ is directly deducible from $\mathbf{u}_{\ell} \approx \mathbf{v}_{\ell}$, there exists some substitution $\varphi: \mathscr{A} \rightarrow T_{\mathscr{A}}$ such that $\mathbf{u}_{\ell} \varphi$ is a subterm of $\mathbf{t}_{\ell}$. 
Lemma 7.6. The identity $\mathbf{u}_{\ell} \approx \mathbf{v}_{\ell}$ cannot be from $\Theta_{\min }$.

Proof. Suppose that $\mathbf{u}_{\ell} \approx \mathbf{v}_{\ell}$ is an identity from (3.1i)-(3.1v). Then,

$$
\mathbf{u}_{\ell}=\mathbf{h}_{0} x^{\otimes_{1}} \mathbf{h}_{1} y^{\circledast_{2}} \mathbf{h}_{2} x^{\circledast_{3}} \mathbf{h}_{3} y^{\otimes_{4}} \mathbf{h}_{4}
$$

for some $\mathbf{h}_{i} \in\left(\mathscr{A} \cup \mathscr{A}^{*}\right)^{+} \cup\{\varnothing\}$ and $\circledast_{i} \in\{1, *\}$. Let $z \in \operatorname{con}(\overline{\lfloor x \varphi\rfloor})$ and $t \in \operatorname{con}(\overline{\lfloor y \varphi\rfloor})$, so that

$$
\left\lfloor\mathbf{u}_{\ell} \varphi\right\rfloor=\left\lfloor\mathbf{h}_{0} \varphi\right\rfloor \underbrace{\left(\cdots z^{\circledast_{1}^{\prime}} \cdots\right)}_{\left\lfloor(x \varphi)^{\oplus_{1}}\right\rfloor}\left\lfloor\mathbf{h}_{1} \varphi\right\rfloor \underbrace{\left(\cdots t^{\circledast_{2}^{\prime}} \cdots\right)}_{\left\lfloor(y \varphi)^{\oplus_{2}}\right\rfloor}\left\lfloor\mathbf{h}_{2} \varphi\right\rfloor \underbrace{\left(\cdots z^{\circledast_{3}^{\prime}} \cdots\right)}_{\left\lfloor(x \varphi)^{\oplus_{3}}\right\rfloor}\left\lfloor\mathbf{h}_{3} \varphi\right\rfloor \underbrace{\left(\cdots t^{\otimes_{4}^{\prime}} \cdots\right)}_{\left\lfloor(y \varphi)^{\oplus_{4}}\right\rfloor}\left\lfloor\mathbf{h}_{4} \varphi\right\rfloor
$$

for some $\circledast_{i}^{\prime} \in\{1, *\}$. Since $\mathbf{u}_{\ell} \varphi$ is a subterm of $\mathbf{t}_{\ell}$, as observed in (iii) of Remark 7.1, either $\left\lfloor\mathbf{u}_{\ell} \varphi\right\rfloor$ or $\left\lfloor\left(\mathbf{u}_{\ell} \varphi\right)^{*}\right\rfloor$ is a factor of the word $\left\lfloor\mathbf{t}_{\ell}\right\rfloor$, whence $\left\lfloor\mathbf{t}_{\ell}\right\rfloor$ contains a factor of the form (7.2c). But this is impossible by (ii) of Lemma 7.5.

If $\mathbf{u}_{\ell} \approx \mathbf{v}_{\ell}$ is an identity from (3.1a)-(3.1h), then a similar argument shows that the word $\left\lfloor\mathbf{t}_{\ell}\right\rfloor$ contains a factor of the form (7.2a) or (7.2b). This is again impossible by (ii) of Lemma 7.5.

Lemma 7.7. The identity $\mathbf{u}_{\ell} \approx \mathbf{v}_{\ell}$ cannot be from $\Omega \backslash\left\{\mathrm{V}_{\mathrm{fix}}^{\uparrow} \approx \mathrm{V}_{\mathrm{fix}}^{\downarrow}\right\}$.

Proof. It suffices to assume that the identity $\mathbf{u}_{\ell} \approx \mathbf{v}_{\ell}$ belongs to $\Omega$ and then show that it is precisely $\mathrm{V}_{\text {fix }}^{\uparrow} \approx \mathrm{V}_{\text {fix }}^{\downarrow}$. Suppose that $\mathbf{u}_{\ell} \approx \mathbf{v}_{\ell}$ is the identity $\mathrm{w}^{\uparrow} \approx \mathrm{W}^{\downarrow}$ in $\Omega$, where $\mathrm{W}=\left(c_{1}, c_{2}, \ldots, c_{n}\right) \in \mathbb{B}_{n}^{=} \cup \mathbb{B}_{n}^{<}$for some $n \geq 2$. Then, either $\mathbf{u}_{\ell} \varphi=\mathrm{W}^{\uparrow} \varphi$ or $\mathbf{u}_{\ell} \varphi=\mathrm{W}^{\downarrow} \varphi$ is a subterm of $\mathbf{t}_{\ell}$; by symmetry, it suffices to assume that

$$
\mathbf{u}_{\ell} \varphi=W^{\uparrow} \varphi=(x \varphi)\left(\prod_{i=1}^{n}\left(\left(y_{i} \varphi\right)\left(h_{i}^{c_{i}} \varphi\right)\left(y_{i} \varphi\right)^{*}\right)\right)(x \varphi)^{*}
$$

is a subterm of $\mathbf{t}_{\ell}$.

Suppose that the word $\lfloor x \varphi\rfloor$ is non-singleton. Then, $\overline{\lfloor x \varphi\rfloor}=\cdots x_{1} x_{2} \cdots$ for some $x_{1}, x_{2} \in \mathscr{A}$. Choose any $q \in \operatorname{con}\left(\overline{\left\lfloor y_{1} \varphi\right\rfloor}\right)$. Then,

$$
\begin{aligned}
\left\lfloor W^{\uparrow} \varphi\right\rfloor & =\lfloor x \varphi\rfloor\left\lfloor y_{1} \varphi\right\rfloor\left\lfloor h_{1}^{c_{1}} \varphi\right\rfloor\left\lfloor\left(y_{1} \varphi\right)^{*}\right\rfloor \cdots\left\lfloor(x \varphi)^{*}\right\rfloor \\
& =\underbrace{\left(\cdots x_{1}^{\otimes_{1}} x_{2}^{\circledast_{2}} \cdots\right)}_{\lfloor x \varphi\rfloor} \underbrace{\left(\cdots q^{\circledast_{3}} \cdots\right)}_{\left\lfloor y_{1} \varphi\right\rfloor}\left\lfloor h_{1}^{c_{1}} \varphi\right\rfloor \underbrace{\left\lfloor\cdots q^{\otimes_{4}} \cdots\right)}_{\left\lfloor\left(y_{1} \varphi\right)^{*}\right\rfloor} \cdots \underbrace{\left(\cdots x_{2}^{\circledast_{5}} x_{1}^{\circledast_{6}} \cdots\right)}_{\left\lfloor(x \varphi)^{*}\right\rfloor}
\end{aligned}
$$

for some $\circledast_{i} \in\{1, *\}$. Since $\mathrm{W}^{\uparrow} \varphi$ is a subterm of $\mathbf{t}_{\ell}$, as observed in (iii) of Remark 7.1, either $\left\lfloor\mathrm{W}^{\uparrow} \varphi\right\rfloor$ or $\left\lfloor\left(\mathrm{W}^{\uparrow} \varphi\right)^{*}\right\rfloor$ is a factor of the word $\left\lfloor\mathbf{t}_{\ell}\right\rfloor$, whence $\left\lfloor\mathbf{t}_{\ell}\right\rfloor$ contains a factor of the form (7.2b) or (7.2d), depending on whether or not the letters $x_{1}, x_{2}$, and $q$ are distinct. But this is impossible by (ii) of Lemma 7.5. Therefore,

(A) the word $\lfloor x \varphi\rfloor$ is a singleton.

Suppose that the word $\left\lfloor y_{i} \varphi\right\rfloor$ is non-singleton. Then, $\overline{\left\lfloor y_{i} \varphi\right\rfloor}=\cdots x_{1} x_{2} \cdots$ for some $x_{1}, x_{2} \in \mathscr{A}$. Choose any $q \in \operatorname{con}(\overline{\lfloor x \varphi\rfloor})$. Then,

$$
\begin{aligned}
\left\lfloor\mathrm{W}^{\uparrow} \varphi\right\rfloor & =\lfloor x \varphi\rfloor \cdots\left\lfloor y_{i} \varphi\right\rfloor\left\lfloor h_{i}^{c_{i}} \varphi\right\rfloor\left\lfloor\left(y_{i} \varphi\right)^{*}\right\rfloor \cdots\left\lfloor(x \varphi)^{*}\right\rfloor \\
& =\underbrace{\left(\cdots q^{\otimes_{1}} \cdots\right)}_{\lfloor x \varphi\rfloor} \cdots \underbrace{\left(\cdots x_{1}^{\otimes_{2}} x_{2}^{\otimes_{3}} \cdots\right)}_{\left\lfloor y_{i} \varphi\right\rfloor}\left\lfloor h_{i}^{c_{i}} \varphi\right\rfloor \underbrace{\left(\cdots x_{2}^{\otimes_{4}} x_{1}^{\otimes_{5}} \cdots\right)}_{\left\lfloor\left(y_{i} \varphi\right)^{*}\right\rfloor} \cdots \underbrace{\left(\cdots q^{\circledast_{6}} \cdots\right)}_{\left\lfloor(x \varphi)^{*}\right\rfloor}
\end{aligned}
$$

for some $\circledast_{j} \in\{1, *\}$. Since $\mathrm{W}^{\uparrow} \varphi$ is a subterm of $\mathbf{t}_{\ell}$, by (iii) of Remark 7.1, either $\left\lfloor\mathrm{W}^{\uparrow} \varphi\right\rfloor$ or $\left\lfloor\left(\mathrm{W}^{\uparrow} \varphi\right)^{*}\right\rfloor$ is a factor of the word $\left\lfloor\mathbf{t}_{\ell}\right\rfloor$, whence $\left\lfloor\mathbf{t}_{\ell}\right\rfloor$ contains a factor of the form (7.2b) or (7.2d), depending on whether or not the letters $x_{1}$, $x_{2}$, and $q$ are distinct. But this is impossible by (ii) of Lemma 7.5. Therefore,

(B) the word $\left\lfloor y_{i} \varphi\right\rfloor$ is a singleton.

Suppose that $c_{i}=1$ and $\left\lfloor h_{i}^{c_{i}} \varphi\right\rfloor$ is non-singleton. Then, $\overline{\left\lfloor h_{i}^{c_{i}} \varphi\right\rfloor}=\cdots x_{1} x_{2} \cdots$ for some $x_{1}, x_{2} \in \mathscr{A}$. Choose any $q \in \operatorname{con}(\overline{\lfloor x \varphi\rfloor})$ and $t \in \operatorname{con}\left(\overline{\left\lfloor y_{i} \varphi\right\rfloor}\right)$. Then,

$$
\begin{aligned}
\left\lfloor\mathrm{W}^{\uparrow} \varphi\right\rfloor & =\lfloor x \varphi\rfloor \cdots\left\lfloor y_{i} \varphi\right\rfloor\left\lfloor h_{i}^{c_{i}} \varphi\right\rfloor\left\lfloor\left(y_{i} \varphi\right)^{*}\right\rfloor \cdots\left\lfloor(x \varphi)^{*}\right\rfloor \\
& =\underbrace{\left(\cdots q^{\circledast_{1}} \cdots\right)}_{\lfloor x \varphi\rfloor} \cdots \underbrace{\left(\cdots t^{\circledast_{2}} \cdots\right)}_{\left\lfloor y_{i} \varphi\right\rfloor} \underbrace{\left(\cdots x_{1}^{\circledast_{3}} x_{2}^{\circledast_{4}} \cdots\right)}_{\left\lfloor h_{i}^{c_{i}} \varphi\right\rfloor} \underbrace{\left(\cdots t^{\circledast_{5}} \cdots\right)}_{\left\lfloor\left(y_{i} \varphi\right)^{*}\right\rfloor} \cdots \underbrace{\left(\cdots q^{\circledast_{6}} \cdots\right)}_{\left\lfloor(x \varphi)^{*}\right\rfloor}
\end{aligned}
$$

for some $\circledast_{j} \in\{1, *\}$. Since $\mathrm{W}^{\uparrow} \varphi$ is a subterm of $\mathbf{t}_{\ell}$, by (iii) of Remark 7.1, either $\left\lfloor\mathrm{W}^{\uparrow} \varphi\right\rfloor$ or $\left\lfloor\left(\mathrm{W}^{\uparrow} \varphi\right)^{*}\right\rfloor$ is a factor of the word $\left\lfloor\mathbf{t}_{\ell}\right\rfloor$, whence $\left\lfloor\mathbf{t}_{\ell}\right\rfloor$ contains a subterm of the form (7.2b), (7.2d), or (7.2e), depending on whether or not the letters $x_{1}, x_{2}, q$, and $t$ are distinct. But this is impossible by (ii) of Lemma 7.5. Therefore, (C) if $c_{i}=1$, then the word $\left\lfloor h_{i}^{c_{i}} \varphi\right\rfloor$ is a singleton. 
Now since $\mathbf{u}_{\ell} \varphi=\mathrm{W}^{\uparrow} \varphi$ is a subterm of $\mathbf{t}_{\ell}$, by (iii) of Remark 7.1, either $\left\lfloor\mathrm{W}^{\uparrow} \varphi\right\rfloor$ or $\left\lfloor\left(\mathrm{W}^{\uparrow} \varphi\right)^{*}\right\rfloor$ is a factor of the word $\left\lfloor\mathbf{t}_{\ell}\right\rfloor=\mathrm{v}_{\text {fix }}^{\uparrow}$, where

$$
\left\lfloor W^{\uparrow} \varphi\right\rfloor=\lfloor x \varphi\rfloor\left(\prod_{i=1}^{n}\left(\left\lfloor y_{i} \varphi\right\rfloor\left\lfloor h_{i}^{c_{i}} \varphi\right\rfloor\left\lfloor\left(y_{i} \varphi\right)^{*}\right\rfloor\right)\right)\left\lfloor(x \varphi)^{*}\right\rfloor
$$

and

$$
\left\lfloor\left(W^{\uparrow} \varphi\right)^{*}\right\rfloor=\lfloor x \varphi\rfloor\left(\prod_{i=n}^{1}\left(\left\lfloor y_{i} \varphi\right\rfloor\left\lfloor\left(h_{i}^{c_{i}} \varphi\right)^{*}\right\rfloor\left\lfloor\left(y_{i} \varphi\right)^{*}\right\rfloor\right)\right)\left\lfloor(x \varphi)^{*}\right\rfloor .
$$

Therefore, (A)-(C) imply that $m=n$ and either $\mathrm{v}_{\text {fix }}^{\uparrow}=\left\lfloor\mathrm{W}^{\uparrow} \varphi\right\rfloor$ or $\mathrm{V}_{\text {fix }}^{\uparrow}=\left\lfloor\left(\mathrm{W}^{\uparrow} \varphi\right)^{*}\right\rfloor$. It follows that $\mathrm{v}_{\text {fix }}^{\uparrow} \approx \mathrm{V}_{\text {fix }}^{\downarrow}$ coincides with the identity $\mathrm{w}^{\uparrow} \approx \mathrm{W}^{\downarrow}$ and so also the identity $\mathbf{u}_{\ell} \approx \mathbf{v}_{\ell}$.

Acknowledgement: The author would like to thank the anonymous referees for helpful suggestions, and Igor Dolinka and Marcel G. Jackson for very fruitful discussions.

\section{References}

[1] Y. A. Bahturin and A. Y. Ol'shanskiĬ, Identical relations in finite Lie rings, Math. USSR Sb. 25 (1975), 507-523.

[2] G. Birkhoff, On the structure of abstract algebra, Proc. Cambridge Philos. Soc. 31 (1935), 433-454.

[3] J. Hage and T. Harju, On involutions arising from graphs, in: Algorithmic Bioprocesses, Nat. Comput. Ser., Springer, Berlin (2009), 623-630.

[4] M. Jackson, Finite semigroups with infinite irredundant identity bases, Internat. J. Algebra Comput. 15 (2005), 405-422.

[5] J. Ježek, Nonfinitely based three-element idempotent groupoids, Algebra Universalis 20 (1985), 292-301.

[6] J. Kad'ourek, On varieties of combinatorial inverse semigroups. II, Semigroup Forum 44 (1992), 53-78.

[7] R. L. Kruse, Identities satisfied by a finite ring, J. Algebra 26 (1973), 298-318.

[8] E. W. H. Lee, A sufficient condition for the non-finite basis property of semigroups, Monatsh. Math. 168 (2012), 461-472.

[9] E. W. H. Lee, A class of finite semigroups without irredundant bases of identities, to appear in Yokohama Math. J.

[10] E. W. H. Lee, Finitely based finite involution semigroups with non-finitely based reducts, to appear in Quaest. Math.

[11] E. W. H. Lee, J. R. Li and W. T. Zhang, Minimal non-finitely based semigroups, Semigroup Forum 85 (2012), 577-580.

[12] I. V. L'vov, Varieties of associative rings. I, Algebra Logic 12 (1973), 150-167.

[13] G. I. MashevitskiĬ, An example of a finite semigroup without an irreducible basis of identities in the class of completely 0-simple semigroups, Russian Math. Surveys 38 (1983), no. 2, 192-193.

[14] R. McKenzie, Tarski's finite basis problem is undecidable, Internat. J. Algebra Comput. 6 (1996), 49-104.

[15] V. L. Murskiĭ, The existence in the three-valued logic of a closed class with a finite basis having no finite complete system of identities, Soviet Math. Dokl. 6 (1965), 1020-1024.

[16] S. Oates and M. B. Powell, Identical relations in finite groups, J. Algebra 1(1964), 11-39.

[17] P. Perkins, Bases for equational theories of semigroups, J. Algebra 11 (1969), 298-314.

[18] M. V. Sapir, On Cross semigroup varieties and related questions, Semigroup Forum 42 (1991), 345-364.

[19] M. V. Volkov, The finite basis problem for finite semigroups, Sci. Math. Jpn. 53 (2001), 171-199.

Received May 29, 2014; revised January 3, 2015. 